\title{
Clinical Challenges in the Management of Hormone Receptor-Positive, Human Epidermal Growth Factor Receptor 2-Negative Metastatic Breast Cancer: A Literature Review
}

\author{
Gayathri Nagaraj (D) C Cynthia X. Ma
}

Received: July 30, 2020 / Accepted: October 24, 2020 / Published online: November 15, 2020

(C) The Author(s) 2020

\section{ABSTRACT}

Endocrine therapy (ET) is integral to the treatment of hormone receptor-positive (HR+), human epidermal growth factor receptor 2-negative (HER2-) metastatic breast cancer (MBC). Aromatase inhibitors (AIs; e.g., anastrozole, letrozole, exemestane), selective estrogen receptor modulators (e.g., tamoxifen), and the selective estrogen receptor degrader, fulvestrant, inhibit tumor cell proliferation by targeting ER signaling. However, the efficacy of ET could be limited by intrinsic and acquired resistance mechanisms, which has prompted the development of targeted agents and combination strategies. In recent years, the treatment landscape for $\mathrm{HR}+$, HER2- MBC has evolved rapidly. AIs, historically the first-line treatment for postmenopausal patients with $\mathrm{HR}+$, HER2 - MBC, have been challenged by more effective ET, such as fulvestrant alone or in combination with an $\mathrm{AI}$, and the cyclin-dependent kinase (CDK)4/6 inhibitors, which have increasingly become the new standard of

G. Nagaraj ( $\square)$

Division of Medical Oncology and Hematology, Loma Linda University School of Medicine, Loma Linda, CA 92350, USA

e-mail: GNagaraj@llu.edu

C. X. Ma

Division of Oncology, Washington University

School of Medicine, St. Louis, MO 63110, USA care. For endocrine-resistant disease ( $\geq$ secondline), clinical trials demonstrated that the mammalian target of rapamycin inhibitor, everolimus, enhanced the efficacy of exemestane or fulvestrant after progression on an AI. CDK4/6 inhibitors in combination with fulvestrant have demonstrated superior progressionfree survival and overall survival versus fulvestrant alone. Recently, the combination of fulvestrant with alpelisib in phosphatidylinositol4,5-bisphosphate 3-kinase (PIK3CA) mutated $\mathrm{HR}+$, HER2- MBC following progression on or after ET was approved, based on the SOLAR-1 study. However, the optimal sequencing of treatments is unknown, especially following disease progression on a CDK4/6 inhibitor. This review aims to provide practical guidance for the management of HR+, HER2- MBC based on available data and the utility of genomic biomarkers, including germline breast cancer genes 1 and 2 (BRCA1/2) mutations, and somatic estrogen receptor alpha gene (ESR1), HER2, and PIK3CA mutations.

Keywords: Aromatase inhibitors; Cyclindependent kinase 4/6 inhibitors; Endocrine therapy; Fulvestrant; Hormone receptorpositive, human epidermal growth factor receptor 2-negative metastatic breast cancer; Mammalian target of rapamycin inhibitors; Phosphoinositide 3-kinase pathway inhibitors; Selective estrogen receptor degrader 


\section{Key Summary Points}

Significant progress has been made in recent years for the treatment of postmenopausal women with hormone receptor-positive $(\mathrm{HR}+)$, human epidermal growth factor receptor 2-negative (HER2-) metastatic breast cancer (MBC).

In the first-line setting, fulvestrant alone or in combination with an aromatase inhibitor (AI) has been shown to be superior to an $\mathrm{AI}$ alone, whereas combinations of endocrine therapy (ET) plus a cyclin-dependent kinase (CDK) $4 / 6$ inhibitor has increasingly become the new standard of care.

Combinations of ET plus CDK4/6 or mammalian target of rapamycin inhibitors have been approved in the ET-resistant setting.

Recent approval of alpelisib with fulvestrant in phosphatidylinositol-4,5bisphosphate 3-kinase (PIK3CA) mutated $\mathrm{HR}+$, HER2 - MBC following progression on or after ET marks the first combination therapy approved based on somatic mutations in MBC.

The role for genomic biomarkers in guiding individualized treatment of $\mathrm{HR}+$, HER2- MBC is expanding.

\section{DIGITAL FEATURES}

This article is published with digital features, including a summary slide, to facilitate understanding of the article. To view digital features for this article go to https://doi.org/10.6084/ m9.figshare.13134812.

\section{INTRODUCTION}

Breast cancer (BC) is commonly categorized into three major subtypes, based on the presence or absence of molecular markers for estrogen or progesterone receptors and human epidermal growth factor 2 (HER2): hormone receptor-positive $(\mathrm{HR}+)$, HER2-positive, and triple-negative (tumors lacking estrogen or progesterone receptors and HER2) [1]. Based on gene expression profiling, the molecular classification of BC includes luminal A and luminal B, HER2-enriched, and basal subtypes. The luminal subtypes make up the majority of HR+ BC [2]. Approximately $75 \%$ of BCs are $\mathrm{HR}+$ $[3,4]$. Endocrine therapy (ET) inhibits estrogen receptor (ER) signaling [5] and plays a fundamental role in the management of HR+ disease by reducing relapse risk and improving survival [6]. Compared with tamoxifen, aromatase inhibitors (AIs) have shown superior efficacy across all stages of BC in both postmenopausal and premenopausal women (in combination with ovarian suppression) and have emerged as key agents for the treatment of $\mathrm{HR}+\mathrm{BC}$ in the last two decades $[6,7]$. AIs used to be the firstline treatment of choice for postmenopausal women with $\mathrm{HR}+$, HER2-negative (HER2-) metastatic BC (MBC). However, cyclin-dependent kinase (CDK)4/6 inhibitors (palbociclib, ribociclib, abemaciclib) in combination with ET [8-10] and more effective ET approaches, such as fulvestrant [11] or fulvestrant in combination with an $\mathrm{AI}$, have now demonstrated improved efficacy $[12,13]$.

The selective estrogen receptor degrader (SERD) fulvestrant binds to and induces rapid degradation of the ER. Unlike the selective ER modulator tamoxifen, which exerts agonist activity in some organs, including the uterus, bone, and cardiovascular system [1], fulvestrant lacks agonistic actions [14]. Though initially approved for the treatment of patients with ETresistant, $\mathrm{HR}+$ advanced $\mathrm{BC}$ (ABC), fulvestrant has subsequently been approved for use in the first-line setting after demonstrating improvements in progression-free survival (PFS) versus the AI, anastrozole $[11,15]$. Fulvestrant has also been shown to retain relative efficacy in the 
presence of some ER alpha gene (ESR1) mutations, albeit with efficacy that is reduced versus wild-type ESR1 [16]. As a result of its unique mechanism of action, fulvestrant has become a common partner for targeted therapy combinations. CDK4/6 inhibitors have been approved in combination with fulvestrant in the first-line (ribociclib) [9] and endocrine-resistant (palbociclib, ribociclib, abemaciclib) settings [8-10]. More recently, fulvestrant has demonstrated efficacy when combined with phosphoinositide 3-kinase (PI3K) inhibitors for the treatment of patients with $\mathrm{HR}+$, HER2-, phosphatidylinositol-4,5-bisphosphate 3-kinase (PIK3CA) mutation-positive $\mathrm{MBC}$ who have progressed on an AI $[17,18]$. The combination of fulvestrant with alpelisib has been approved for use in this setting [19].

As the mechanism of action and clinical utility of various ET agents in $\mathrm{HR}+\mathrm{BC}$ have been reviewed extensively [20], this review will focus on recent progress in optimizing ET and the development of targeted agents in the management of $\mathrm{HR}+\mathrm{MBC}$.

This article is based on previously conducted studies and does not contain any studies with human participants or animals performed by any of the authors.

\section{ET IN THE TREATMENT OF HR+, HER2 - MBC, MONOTHERAPY VERSUS COMBINATION THERAPY- WHAT DO THE DATA TELL US?}

In HR+, HER2- MBC, ET has been used as monotherapy, in dual ER-targeting strategies, and in combination with targeted agents. While AIs have previously demonstrated superiority to tamoxifen in postmenopausal women [21], the SERD fulvestrant, as monotherapy or in combination with an AI, has demonstrated superior efficacy to AIs in ET-naïve patients $[13,15]$.

\section{Fulvestrant as Monotherapy and in Combination with AIs}

Initial studies that led to the approval of fulvestrant by the US Food and Drug
Administration (FDA) in 2002 were based on a 250-mg dose, administered monthly by intramuscular injection [22]. In some studies, a 500-mg loading dose was also administered.

The phase III SWOG S0226 study compared the combination of fulvestrant (initial 500-mg loading dose, followed by a 250-mg dose every 14 days for the first month, and every 28 days thereafter) plus anastrozole versus anastrozole alone in the first-line setting $[12,13]$. Improvements in median PFS [15.0 vs. 13.5 months; hazard ratio for progression or death 0.81 (95\% confidence interval (CI) $0.69-0.94$ ); $P=0.007$ ] and overall survival [OS; 49.8 vs. 42.0 months; hazard ratio 0.82 (95\% CI 0.69-0.98); $P=0.03$ ] were observed for combination therapy versus anastrozole alone [13]. When data were stratified according to prior tamoxifen therapy, improvements in PFS and OS with combination therapy versus anastrozole alone were limited to patients who had not received prior tamoxifen therapy [13]. Subsequently, the phase III CONFIRM study established the superiority of the 500 -mg dose of fulvestrant $(500 \mathrm{mg}$ administered on Days 0,14 , and 28, and every 28 days thereafter) versus the previously approved 250 -mg dose $(250 \mathrm{mg}$ administered on Days 0 and 28 , and every 28 days thereafter) in postmenopausal women with $\mathrm{HR}+\mathrm{MBC}$ who failed previous hormonal therapy, establishing $500 \mathrm{mg}$ as the recommended dose $[11,23]$. In CONFIRM, median PFS was 5.5 and 6.5 months for the 250- and 500-mg doses, respectively [hazard ratio $0.80 \quad(95 \%$ CI $0.68-0.94)$; $P=0.006]$. Median OS was 22.3 months with the 250-mg dose versus 26.4 months with the $500-\mathrm{mg}$ dose [hazard ratio $0.81 \quad(95 \%$ CI 0.69-0.96); nominal $P=0.02]$ [23, 24]. The results of comparative studies of fulvestrant $250 \mathrm{mg}$ (whether used alone or in combination) and other endocrine agents should therefore be interpreted in the context of this dosing change.

The phase II FIRST study compared fulvestrant $(500 \mathrm{mg} / \mathrm{month})$ with the AI anastrozole $(1 \mathrm{mg} /$ day) in postmenopausal women with HR+ locally advanced or MBC not suitable for curative therapy [25]. No previous ET for advanced disease was allowed, although patients could have received ET in the adjuvant 
setting if it had been completed more than 12 months before randomization. The clinical benefit rate at 6 months (primary endpoint) was $72.5 \%$ for patients treated with fulvestrant and $67.0 \%$ for those receiving anastrozole [odds ratio 1.30 (95\% CI $0.72-2.38$ ); $P=0.386$ ] [25]. At follow-up analysis, the median time to progression was 23.4 months for fulvestrant versus 13.1 months for anastrozole [hazard ratio 0.66 (95\% CI 0.47-0.92); $P=0.01$ ] [26], and the final OS analysis demonstrated that median OS was longer with fulvestrant (54.1 months) compared with anastrozole [48.4 months; hazard ratio 0.70 (95\% CI $0.50-0.98) ; P=0.04$ ] [27]. The results of this study were the first to show an efficacy benefit for fulvestrant over anastrozole in the first-line setting.

The phase III FALCON study compared fulvestrant (500 mg administered on Days 0, 14, and 28 , and every 28 days thereafter) with anastrozole (1 mg/day) in postmenopausal women with HR+, HER2 - locally advanced or MBC who had not previously received ET (Table 1) [15]. Treatment with fulvestrant resulted in a significantly longer PFS (primary endpoint) compared with anastrozole [hazard ratio 0.80 (95\% CI 0.637-0.999); $P=0.049$ ]. Median PFS for fulvestrant was 16.6 months (95\% CI 13.83-20.99) versus 13.8 months for anastrozole (95\% CI 11.99-16.59). These findings demonstrated the superior efficacy of fulvestrant versus anastrozole in patients with locally advanced or MBC who had not previously received ET [15]. In August 2017, the FDA approved fulvestrant for the first-line treatment of $\mathrm{HR}+$, HER2 - ABC in postmenopausal women not previously treated with ET [11]. The recent phase II PARSIFAL study, comparing fulvestrant with letrozole used in combination with the CDK4/6 inhibitor palbociclib, showed comparable 4-year OS rates (both 68\%), suggesting that fulvestrant plus palbociclib is a reasonable alternative to letrozole plus palbociclib for the first-line treatment of $\mathrm{HR}+$, HER2 - MBC [28].

\section{ET Combined with CDK4/6 Inhibitors}

One of the hallmarks of cancer is the deregulation of pathways that regulate cell cycle progression [29]. Cell cycle progression from G1 to $S$ phase is restricted by retinoblastoma $(\mathrm{Rb})$ protein via sequestration of E2F family transcriptional factors. CDK4/6, in complex with D-type cyclins, promote $\mathrm{G} 1$ to $\mathrm{S}$ phase progression by the phosphorylation and inactivation of the $\mathrm{Rb}$ protein [30]. ER-positive $(\mathrm{ER}+) \mathrm{BC}$ is particularly dependent on CDK $4 / 6$ activity for cell proliferation, as cyclin D1 is a direct transcriptional target of the ER, as well as other mitogenic signals that mediate endocrine resistance [31]. Additionally, cyclin D1 amplification is a common event in ER+ BC $(58 \%$ in luminal B and 29\% in luminal A cancers) [32], which could lead to constitutive activation of CDK4/6. In preclinical studies, ER+ BCs are particularly sensitive to CDK4/6 inhibitors [33]. There are three CDK4/6 inhibitors currently available for clinical use in combination with an AI or fulvestrant: palbociclib, ribociclib, and abemaciclib [8-10]. Abemaciclib has also been approved by the FDA for use as monotherapy for patients with refractory, heavily pre-treated, HR+, HER2 - MBC, who have progressed on or after prior ET and have received one or two prior chemotherapy regimens for MBC [10]. Approval was based on the results of the phase II, singlearm MONARCH 1 study, which found that $19.7 \%$ of patients achieved an objective response when treated with abemaciclib monotherapy in this setting [34].

Recent phase III studies that have assessed the efficacy of fulvestrant monotherapy and fulvestrant or an AI in combination with targeted therapies, including CDK4/6 inhibitors, for the treatment of postmenopausal patients, are summarized in Table $1[15,18,35-41]$. Table 2 [41-43] lists the phase III studies that have assessed the use of ET in combination with CDK4/6 inhibitors for the treatment of pre- and perimenopausal patients. 


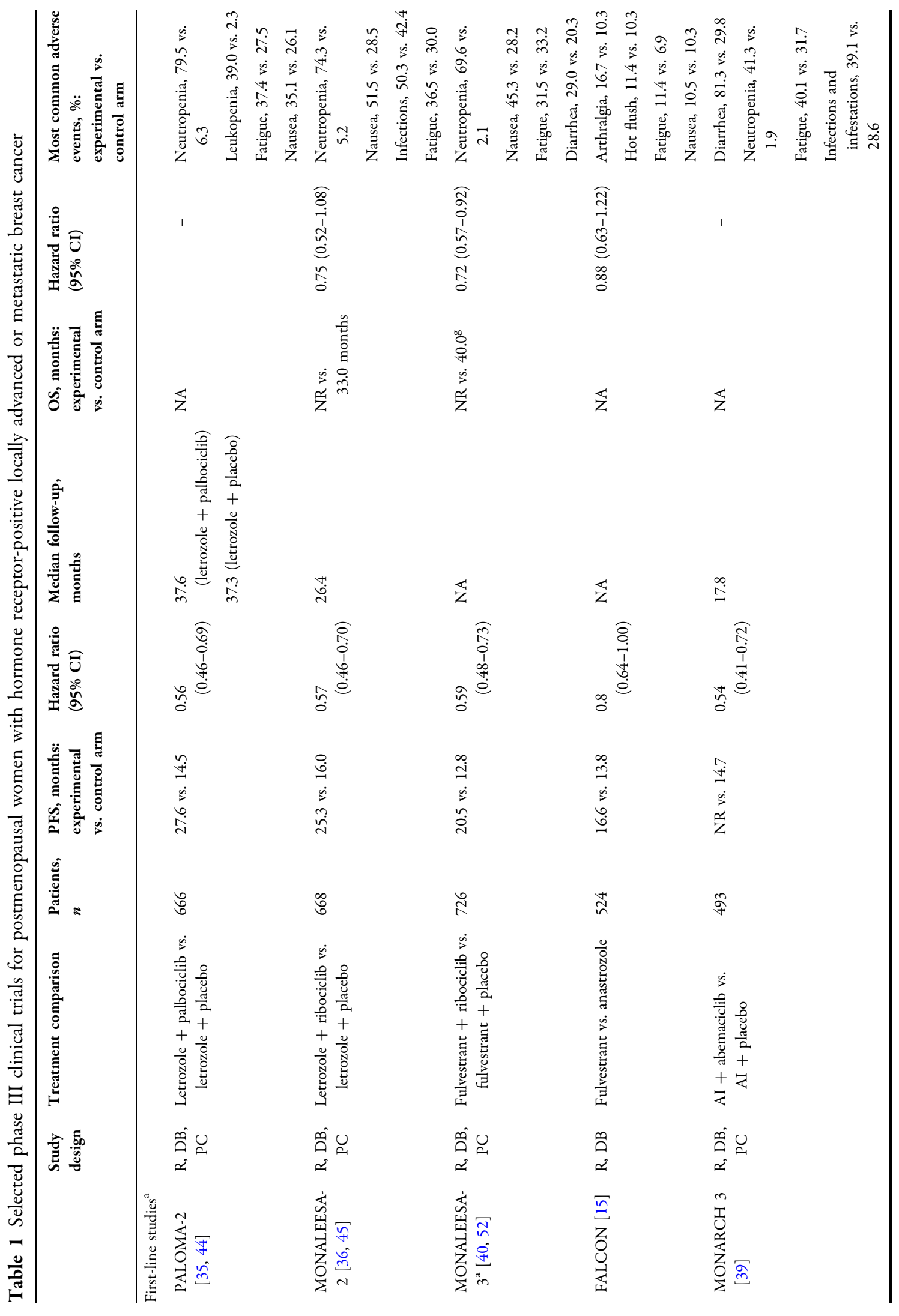




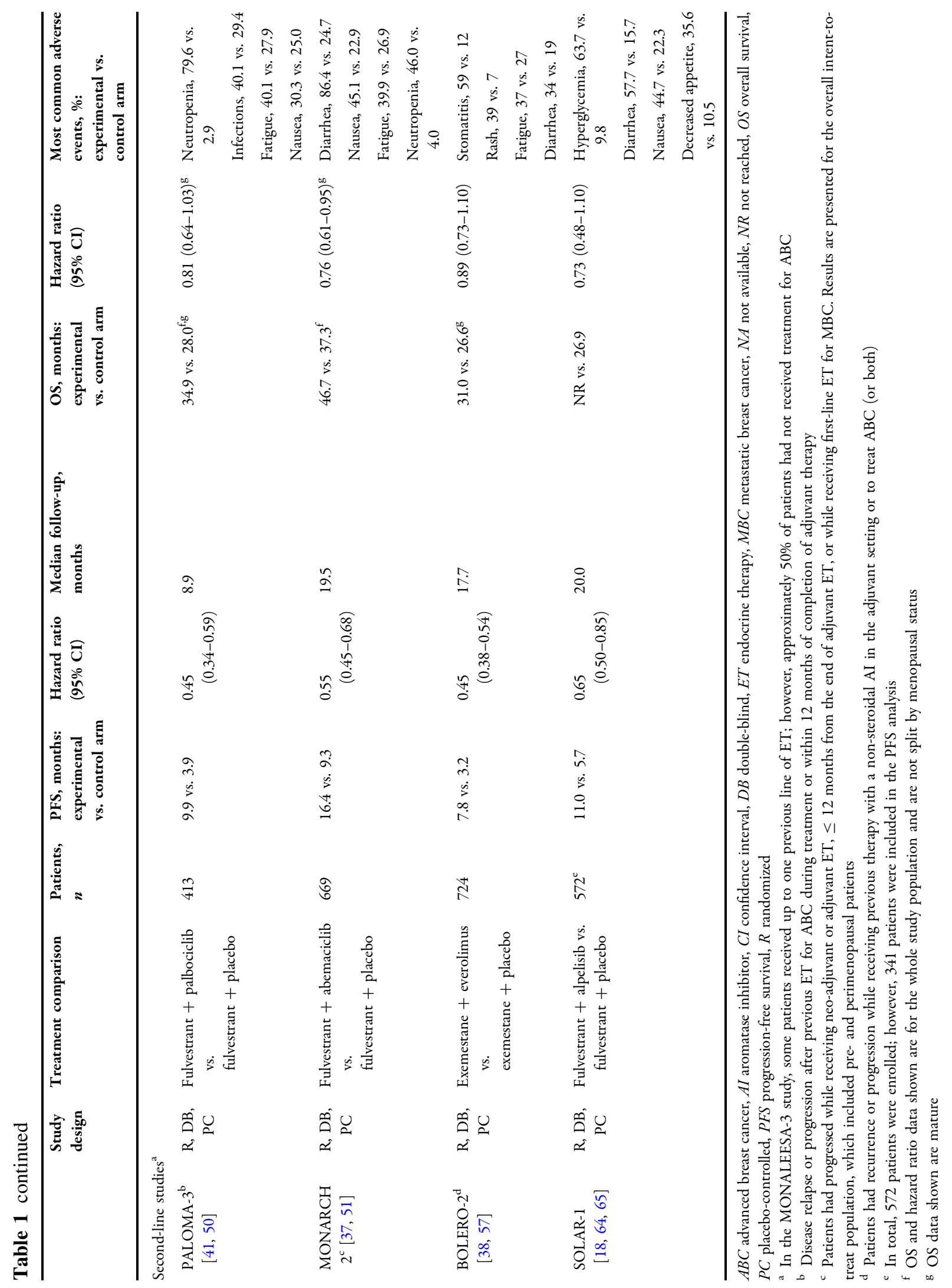




\section{AIs in Combination with CDK4/6 Inhibitors}

CDK4/6 inhibitors have been studied in combination with AIs for the first-line treatment of postmenopausal patients with HR+, HER2locally advanced or MBC (Table 1). In the phase III PALOMA-2 study, median PFS was significantly increased to 27.6 months with palbociclib plus letrozole versus 14.5 months with letrozole monotherapy [hazard ratio for disease progression or death 0.56 (95\% CI 0.46-0.69); $P<0.0001][35,44]$. Similarly, in the phase III MONALEESA-2 study, the combination of ribociclib plus letrozole significantly improved median PFS versus letrozole [25.3 versus 16.0 months; hazard ratio for disease progression or death 0.57 (95\% CI $0.46-0.70)$; $P<0.001][36,45]$. Furthermore, the addition of abemaciclib to anastrozole or letrozole significantly improved median PFS versus anastrozole or letrozole [not reached vs. 14.7 months; hazard ratio for disease progression or death $0.54 \quad(95 \%$ CI $0.41-0.72)$; $P<0.001$ ] in the MONARCH 3 study [39]. Based on these results, the FDA has approved palbociclib, ribociclib, and abemaciclib in combination with an AI for use as first-line treatments for postmenopausal patients with HR+, HER2$\mathrm{ABC}$ or MBC [8-10]. Ribociclib in combination with an AI has also been approved as a first-line treatment for pre- and perimenopausal patients with HR+, HER2- ABC or MBC [9], based on the results of the phase III MONALEESA-7 study (Table 2), which demonstrated significant improvements in median PFS with the addition of ribociclib to ET (anastrozole, letrozole, or tamoxifen plus goserelin) versus ET alone [23.8 vs. 13.0 months; hazard ratio for disease progression or death 0.55 (95\% CI 0.44-0.69); $P<0.0001$ ] [42]. In a protocol-specified second interim analysis, OS was shown to be significantly prolonged in the ET plus ribociclib group compared with ET plus placebo. The estimated OS at 42 months was $70.2 \%$ (95\% CI 63.5-76.0) in the ribociclib group and $46.0 \%$ (95\% CI 32.0-58.9) in the placebo group [hazard ratio for death 0.71 (95\% CI 0.54-0.95); $P=0.00973$ ] (Table 2) [46].

The survival benefit in the subgroup of patients receiving a non-steroidal AI was consistent with the intention-to-treat population.
Notably, MONALEESA-7 is the first study to show the survival benefit of combining a CDK4/6 inhibitor with ET in the first-line setting in HR+, HER2 - ABC in premenopausal women, as previous studies have all been conducted in postmenopausal women [35, 39, 45, 47, 48].

\section{Fulvestrant in Combination with CDK4/6 Inhibitors}

Fulvestrant has been studied in combination with CDK4/6 inhibitors in both the first- and second-line settings, as summarized in Table 1 (postmenopausal women) and Table 2 (pre- and perimenopausal women). Three separate phase III trials in patients with $\mathrm{HR}+$, HER2 - ABC have assessed fulvestrant in combination with the CDK4/6 inhibitors palbociclib (PALOMA-3), ribociclib (MONALEESA-3), and abemaciclib (MONARCH 2) [37, 40, 49]. Results from these studies demonstrated that treatment with each of the CDK4/6 inhibitors in combination with fulvestrant significantly prolonged PFS versus fulvestrant alone (Tables 1, 2) [37, 40, 49]. As a result, the FDA has approved fulvestrant in combination with palbociclib, ribociclib, and abemaciclib for patients with HR+, HER2- ABC or MBC [8-10]. In the OS analysis of PALOMA-3, median OS was not significantly improved with fulvestrant plus palbociclib (34.9 months) versus fulvestrant monotherapy [28.0 months; hazard ratio 0.81 (95\% CI 0.64-1.03); $P=0.09$ ] in the overall patient population (Table 1) [50]. Despite this, in patients with sensitivity to prior ET, improvements in median OS were observed with fulvestrant plus palbociclib (39.7 months) versus fulvestrant monotherapy [29.7 months; hazard ratio 0.72 (95\% CI 0.55-0.94)], suggesting that combination therapy may offer a survival benefit in this patient subgroup [50]. In the subsequent OS analysis of MONARCH 2, fulvestrant in combination with abemaciclib significantly prolonged median OS (46.7 months) versus fulvestrant alone [37.3 months; hazard ratio $0.76 \quad(95 \%$ CI 0.61-0.95); $P=0.01$ ] (Table 1) [51]. Similarly, in MONALEESA-3, fulvestrant in combination with ribociclib had a significant OS benefit over fulvestrant alone [median oS not reached vs. 40.0 months, respectively; hazard ratio 0.72 (95\% CI 0.57-0.92); $P<0.005$ ] (Table 1) [52]. In 


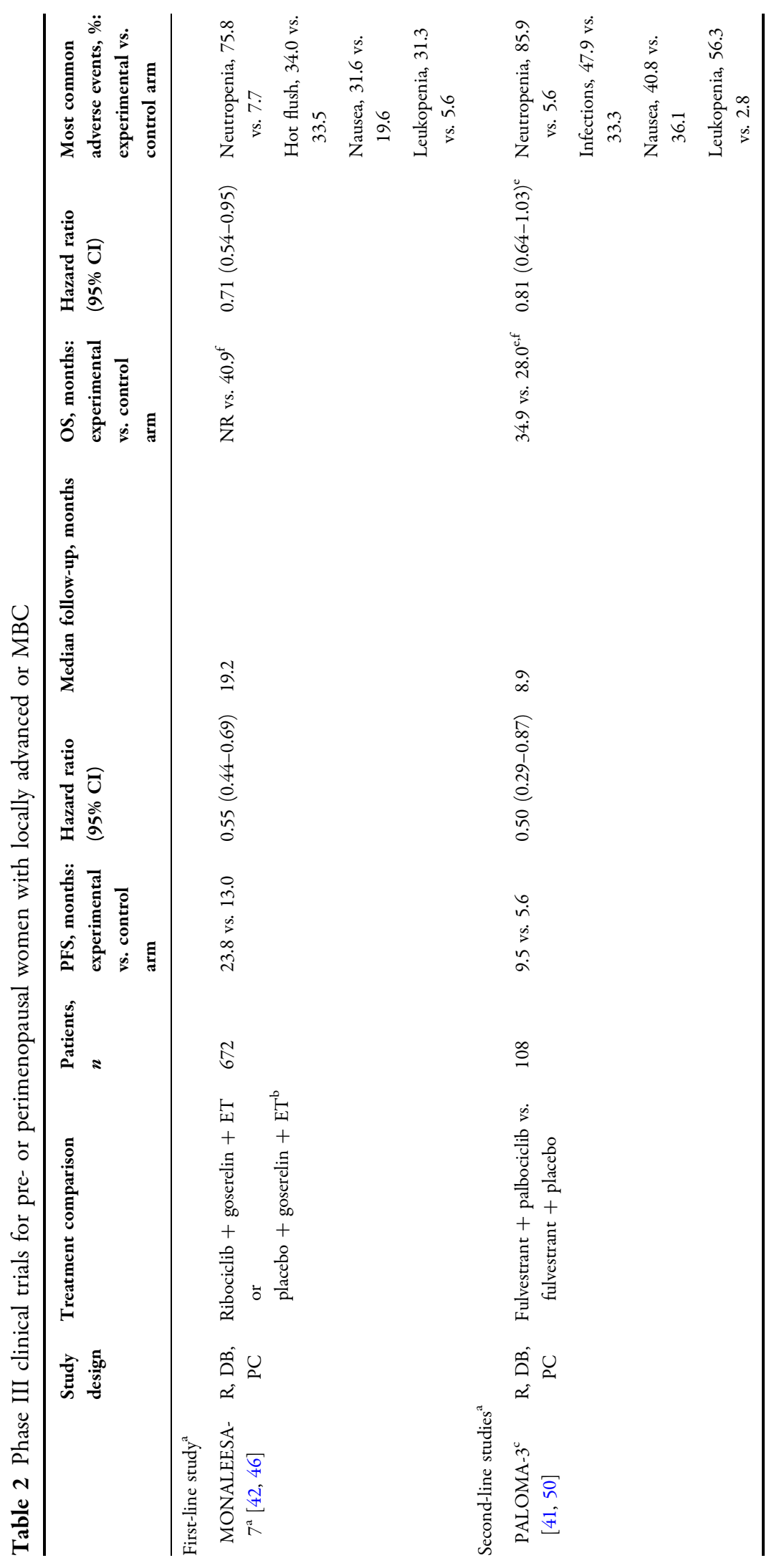




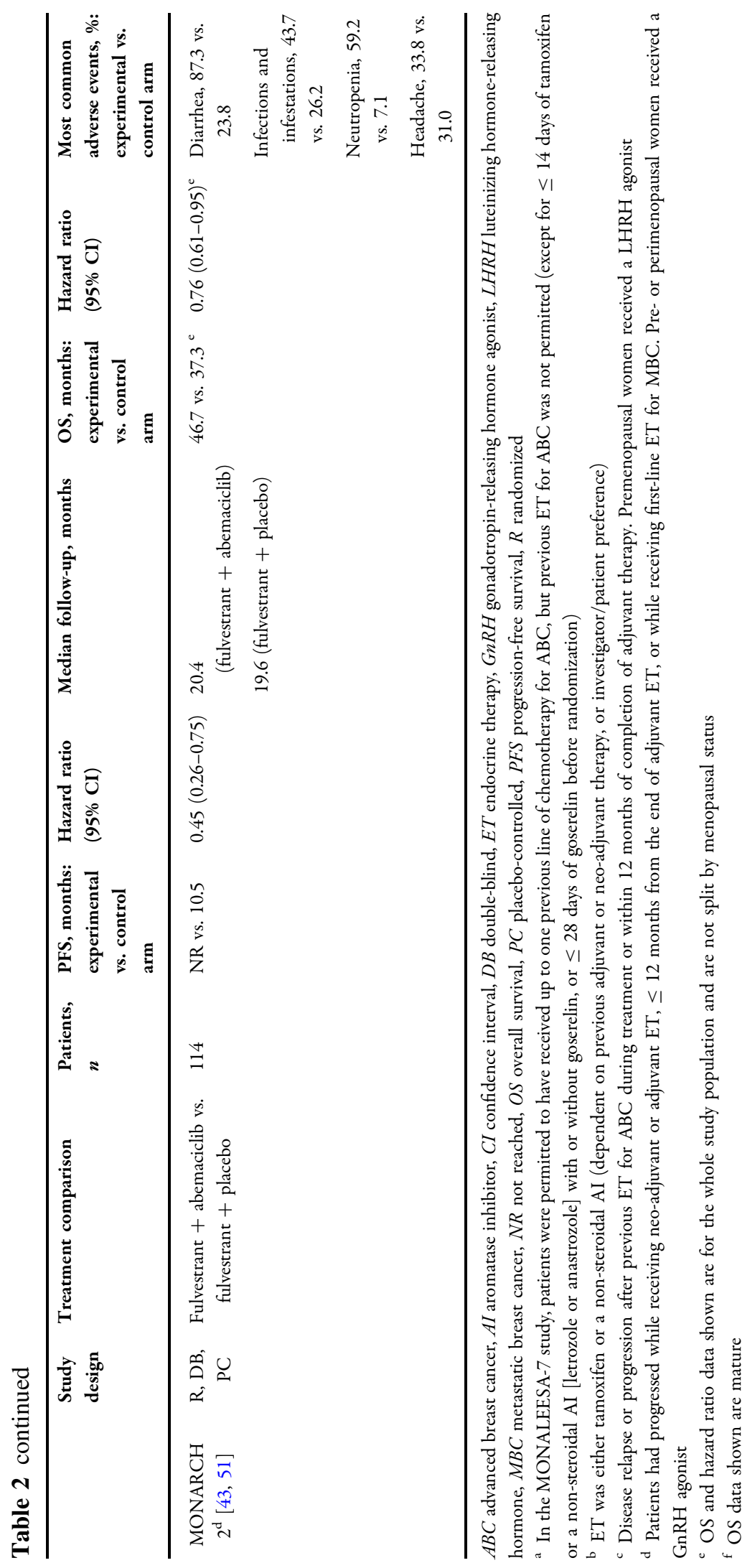


MONARCH 2 and MONALEESA-3, the OS benefit of combination therapy was largely consistent across subgroups analyzed [51, 52].

In all three studies, randomization was stratified by metastatic site, and approximately $60 \%$ of patients with HR+, HER2- ABC had visceral metastases at baseline [37, 40, 49]. In PALOMA-3, PFS was prolonged with the combination of palbociclib plus fulvestrant versus fulvestrant alone in the subgroups of patients with visceral metastases [hazard ratio 0.47 (95\% CI 0.34-0.63)] or with non-visceral metastases [hazard ratio 0.43 (95\% CI 0.28-0.67)] [49]. Similar results were reported in MONARCH 2, where median PFS was improved with the combination of fulvestrant plus abemaciclib versus fulvestrant alone in the subgroups of patients with visceral metastases [hazard ratio 0.48 (95\% CI 0.37-0.63)] or with bone metastases [hazard ratio 0.54 (95\% CI 0.36-0.83)], and the metastatic site subgroup did not have a statistically significant interaction with PFS $(P=0.171)$ [37]. These hazard ratios were maintained with updated PFS data at the next interim analysis [visceral metastases, hazard ratio 0.47 (95\% CI 0.37-0.60); bone metastases, hazard ratio 0.58 (95\% CI 0.40-0.84)] [51].

Results from the MONALEESA-3 study also demonstrated superior PFS with the combination of ribociclib plus fulvestrant, compared with fulvestrant alone, in the subgroup of patients with liver or lung metastases [hazard ratio 0.65 (95\% CI 0.48-0.86)] and in the subgroup of patients without liver or lung metastases [hazard ratio 0.56 (95\% CI $0.42-0.76)$ ] [40]. The PFS results from these subgroups of patients were therefore consistent with the improved PFS observed with the combined treatment of a CDK4/6 inhibitor plus fulvestrant versus fulvestrant alone in the overall patient population for these three studies $[37,40,49]$.

The combination of fulvestrant with palbociclib or abemaciclib has also been examined in pre- and perimenopausal women with $\mathrm{HR}+$, HER2 - ABC in studies that used ovarian suppression (Table 2) [37, 49]. Results from the PALOMA-3 study showed that the addition of palbociclib to fulvestrant improved PFS in the pre- and perimenopausal patient subgroup [hazard ratio 0.50 (95\% CI 0.29-0.87)], and improvements were comparable in the postmenopausal patient subgroup [hazard ratio 0.45 (95\% CI 0.34-0.59)] [49]. In the MONARCH 2 study, menopausal status was not found to have a significant impact on PFS $(P=0.25)$. Treatment with fulvestrant plus abemaciclib prolonged PFS versus fulvestrant alone in both the pre- and perimenopausal patient subgroup [hazard ratio 0.42 (95\% CI 0.25-0.70)] and the postmenopausal patient subgroup [hazard ratio 0.58 (95\% CI 0.46-0.73)] [37]. Fulvestrant plus abemaciclib prolonged OS versus fulvestrant alone in both the pre- and perimenopausal patient subgroups [hazard ratio 0.69 (95\% CI 0.38-1.25)] and the postmenopausal patient subgroup [hazard ratio 0.77 (95\% CI 0.61-0.98)] [51].

\section{Tolerability of CDK4/6 Inhibitors in Combination with ET}

Neutropenia is the most common adverse event with both palbociclib and ribociclib when used in combination with ET [37, 40, 41]. Neutropenia was reported in $79.6 \%$ (grade $3 / 4$ : $61.7 \%$ ) of postmenopausal patients who received palbociclib in PALOMA-3 and $69.6 \%$ (grade 3/4: 53.4\%) of patients who received ribociclib in MONALEESA-3 [40, 41]. By comparison, the incidence of neutropenia in postmenopausal women was lower with abemaciclib in MONARCH 2 (46.0\%; grade 3/4: 26.5\%) [37]. Although the risk of febrile neutropenia and neutropenic sepsis was low (approximately 1\%), given the frequency of neutropenia, the FDA recommends monitoring complete blood count before and during the initial months of, treatment with any of the three CDK4/6 inhibitors [8-10]. By comparison, the incidence of diarrhea was much higher with abemaciclib [86.4\% in MONARCH 2 (and $87.1 \%$ at a subsequent follow-up)] than with palbociclib $(21.4 \%$ in PALOMA-3; $20.1 \%$ in postmenopausal patients only) and ribociclib (29.0\% in MONALEESA-3) $[37,40,41,49,51]$, which is reflected by the FDA warning to instruct patients receiving abemaciclib to monitor for diarrhea, and to initiate antidiarrheal therapy and notify healthcare providers at the first sign of diarrhea [10]. Similarly, based on the increased incidence 
of grade $\geq 3$ elevations in alanine aminotransferase and aspartate aminotransferase observed with ribociclib and abemaciclib [37, 40], the FDA also recommends performing liver function tests to monitor increases in serum transaminase levels before initiating, and during the initial months of treatment with ribociclib or abemaciclib [9, 10]. The FDA also recommends monitoring electrocardiograms for QT interval prolongation before initiation of, and every 2 weeks during, the first month of treatment with ribociclib, and monitoring patients for signs of venous thromboembolism during treatment with abemaciclib $[9,10]$.

\section{ET in Combination with Inhibitors of the PI3K-AKT-mTOR Pathway}

The PI3K-AKT-mammalian target of rapamycin (mTOR) pathway is a crucial signaling pathway that regulates important cellular functions, such as growth, proliferation, differentiation, and survival [53], and there is notable crosstalk among genes within the PI3K pathway and external crosstalk with ER activation/signaling [54]. Components of the PI3K pathway are frequently mutated in BC [54]. Activation of this signaling pathway has been associated with the development of resistance to ET in HR+ BC [55]. Synthetic lethality observed in in vitro studies combining ET with inhibitors of the PI3K pathway has provided the rationale for the clinical development of various inhibitors of this pathway for use in combination with ET [55].

\section{ET Combined with mTOR Inhibitors}

In 2012, the FDA approved the combination of the mTOR inhibitor everolimus plus exemestane for the treatment of patients with HR+ MBC who have progressed on prior treatment with an AI [56]. In the phase III BOLERO-2 study (Table 1), the combination of everolimus plus exemestane showed a statistically significant PFS of 7.8 months versus 3.2 months with exemestane monotherapy [hazard ratio 0.45 (95\% CI 0.38-0.54); $P<0.0001]$, which was the primary endpoint [38]. However, in a follow-up analysis, the secondary endpoint of median OS in patients receiving everolimus plus exemestane was reported to be 31.0 months (95\% CI 28.0-34.6) versus 26.6 months (95\% CI 22.6-33.1) in patients receiving exemestane monotherapy [hazard ratio $0.89 \quad(95 \%$ CI 0.73-1.10); $P=0.14$ ] [57]. Hence, the addition of everolimus did not have an OS benefit. Adverse events were more commonly reported with everolimus plus exemestane versus exemestane monotherapy; the most common grade 3 or 4 adverse events with combination therapy were stomatitis, fatigue, dyspnea, anemia, hyperglycemia, pneumonitis, and elevated gamma-glutamyltransferase [38]. A frequently encountered side effect of mTOR inhibitors such as everolimus is stomatitis. Recent data from the SWISH study demonstrated that the use of prophylactic dexamethasone mouthwash significantly reduced the incidence and severity of all-grade stomatitis in patients receiving everolimus plus exemestane, suggesting that prophylactic use of dexamethasone mouthwash could be a new standard of oral care for patients receiving everolimus and exemestane [58].

In the phase II PrECOG 0102 study (NCT01797120), fulvestrant $500 \mathrm{mg}$ was studied in combination with everolimus for the treatment of postmenopausal women with $\mathrm{HR}+$, HER2 - MBC who have progressed on either steroidal or non-steroidal AI therapy. Median PFS significantly favored combination therapy (10.3 months) versus fulvestrant monotherapy [5.1 months; hazard ratio $0.61 \quad(95 \%$ CI 0.40-0.92); $P=0.02$ ] [59]. Adverse events occurred more frequently with fulvestrant plus everolimus versus fulvestrant monotherapy; the most commonly reported treatment-related adverse events (any grade) were oral mucositis $(53 \%$ vs. $12 \%)$, fatigue ( $42 \%$ vs. $22 \%)$, rash $(38 \%$ vs. $5 \%)$, and anemia (31\% vs. $6 \%$ ) [59].

\section{ET Combined with PI3K Inhibitors}

Several PI3K inhibitors have been assessed in combination with ET and have shown promise in the AI-resistant setting. In the phase II FERGI study, the PI3K inhibitor pictilisib was administered in combination with fulvestrant for the treatment of postmenopausal women with ER+, HER2 - MBC who were resistant to an AI; however, the addition of pictilisib to fulvestrant 
was poorly tolerated and did not significantly improve PFS [60]. In contrast, in the phase III BELLE- 2 and BELLE-3 studies, which assessed the addition of buparlisib to fulvestrant in the AI-resistant setting, median PFS was significantly longer with buparlisib plus fulvestrant versus fulvestrant alone [61, 62]. Although this treatment combination was efficacious in postmenopausal women with ET-resistant, $\mathrm{HR}+$, HER2 - MBC, it was also associated with significant toxicity [61, 62]. It has consequently been suggested that more selective (i.e., alpha-specific) PI3K inhibitors may offer a superior safety profile for use in this setting $[60,61]$.

Fulvestrant in combination with taselisib, a potent beta-sparing PI3K inhibitor, has shown promising activity in patients with $\mathrm{HR}+$, HER2-, PIK3CA mutation-positive MBC [63]. The SANDPIPER study compared fulvestrant (500 mg/month) plus taselisib (4 mg/day) versus fulvestrant alone in postmenopausal women with ER+, HER2-, PIK3CA mutation-positive locally advanced or MBC who experienced disease progression or recurrence during or after treatment with an AI. The combination of fulvestrant plus taselisib significantly improved investigator-assessed PFS compared with fulvestrant monotherapy [hazard ratio 0.70 (95\% CI 0.56-0.89); $P=0.0037]$. Median PFS for the combination therapy was 7.4 months $(95 \% \mathrm{CI}$ 7.26-9.07) versus 5.4 months (95\% CI 3.68-7.29) with fulvestrant monotherapy [17]. However, adverse events were more frequently reported with fulvestrant plus taselisib versus fulvestrant alone; the most common adverse events were diarrhea (60.1\% vs. $19.7 \%)$, hyperglycemia (40.4\% vs. $9.4 \%)$, nausea $(34.1 \%$ vs. $24.4 \%)$, and decreased appetite $(26.4 \%$ vs. $10.3 \%$ ). Approximately $31 \%$ of patients who received fulvestrant plus taselisib experienced grade $\geq 3$ adverse events associated with PI3K inhibition (including diarrhea, colitis, pneumonitis, rash, stomatitis, and hyperglycemia), limiting its clinical application [17].

The phase III SOLAR-1 study (Table 1) [64], evaluating the addition of the alpha-specific PI3K inhibitor alpelisib to fulvestrant versus fulvestrant monotherapy, has recently met its primary endpoint, demonstrating a significant improvement in PFS in patients with $\mathrm{HR}+$, HER2-, PIK3CA mutation-positive ABC that progressed on, or following treatment with, an AI (with or without a CDK4/6 inhibitor) [65]. Median PFS was 11.0 months with alpelisib plus fulvestrant and 5.7 months with fulvestrant monotherapy [hazard ratio for progression or death 0.65 (95\% CI $0.50-0.85) ; \quad P<0.001]$. Clinical benefit was also greater with alpelisib plus fulvestrant versus fulvestrant monotherapy (61.0\% vs. $45.3 \%$, respectively). For the primary endpoint, PIK3CA mutation status was determined using fresh or archival tumor tissues [65]. Adverse events (any grade) were more frequently reported with alpelisib plus fulvestrant versus fulvestrant monotherapy. In the overall population, the most common adverse events that occurred in at least $35 \%$ of patients in either the alpelisib plus fulvestrant group or the fulvestrant monotherapy group were hyperglycemia (63.7\% vs. 9.8\%, respectively), diarrhea $(57.7 \%$ vs. $15.7 \%)$, nausea $(44.7 \%$ vs. $22.3 \%)$, decreased appetite (35.6\% vs. $10.5 \%)$, and rash (35.6\% vs. 5.9\%) [65]. This has led to the FDA approval of this combination along with the approval of companion diagnostics to select patients who have PIK3CA mutations in tumor tissue specimens and/or in circulating tumor DNA (ctDNA) [19, 64].

\section{ET Combined with AKT Inhibitors}

Currently, combining ET with AKT inhibitors is an investigational approach to the treatment of HR+, HER2- MBC. The phase II randomized, double-blind, placebo-controlled FAKTION trial evaluated capivasertib, which is an oral small molecule inhibitor of AKT, in combination with fulvestrant versus placebo combined with fulvestrant in patients with $\mathrm{HR}+, \mathrm{HER} 2-\mathrm{MBC}$ who had progressed on prior AI. In the intention-to-treat population, median PFS was 10.3 months for capivasertib versus 4.8 months for placebo [hazard ratio $0.57 \quad(95 \%$ CI 0.39-0.84); $P=0.0035]$. Median OS was 26 months for capivasertib versus 20 months for placebo [hazard ratio 0.59 (95\% CI 0.34-1.05); 
$P=0.071]$ [66]. In the capivasertib group, notable adverse events included diarrhea, rash, hyperglycemia, and infections. Approximately one-third of the patients in the capivasertib group required dose reduction, which was primarily due to rash and diarrhea. In this study, PIK3CA hotspot mutation or PTEN null by immunohistochemistry did not affect sensitivity to capivasertib. Additional comprehensive genomic analyses of tumors are ongoing to ascertain biomarkers of response to therapy [66].

\section{Treatment Options After Progression on ET Combined with a CDK4/6 Inhibitor}

There are limited data regarding the efficacy of different treatment regimens following disease progression on a CDK4/6 inhibitor with ET. If available, we highly recommend clinical trial options in this setting. Tumor DNA sequencing, either tissue-based or ctDNA-based, for predictive biomarkers, such as PIK3CA mutations, neurotrophic receptor tyrosine kinase (NTRK) gene fusions, and mismatch repair (MMR)-deficiencies should be considered in addition to germline $B R C A$ testing, if not done previously.

Table 3 [67-88] outlines some of the recently reported and ongoing studies employing strategies to tackle resistance with combination therapies in HR+, HER2- MBC. Furthermore, several oral SERDs are also in clinical development, both as monotherapy and in combination with other therapies [73, 76, 77].

The optimal treatment for patients following disease progression on a CDK4/6 inhibitor plus an AI or fulvestrant is unknown; however, several studies are planned or ongoing [70, 89]. For patients who had disease progression while receiving an $\mathrm{AI}$ in combination with a CDK4/6 inhibitor, in the absence of visceral crisis (severe organ dysfunction and rapid progression of disease [90)], there are several potential treatment options before consideration of chemotherapy, including an additional line of ET, such as fulvestrant, ET in combination with other targeted agents (e.g., everolimus plus exemestane) $[38,91]$, or fulvestrant plus alpelisib in PIK3CA mutant tumors [19]. Studies are ongoing to evaluate the role of continued CDK4/6 inhibition in combination with an alternate ET, such as fulvestrant, if an AI was used in combination with a CDK4/6 inhibitor initially [92]. Until the results of these trials are available, such an approach is considered investigational. The combination of everolimus plus exemestane was approved for AI-resistant disease based on the phase III BOLERO-2 study [38); however, its efficacy following CDK4/6 inhibition is not clear. In a retrospective, twoinstitute review of patients with HR+, HER2MBC $(n=41)$ who were treated with everolimus-based combinations after progression on palbociclib-based therapy, modest PFS [median 4.2 months (95\% CI 3.2-6.2)], OS [median 18.7 months (95\% CI 9.5-not reached)], and objective response rate (ORR; 17.1\%) were observed [93]. In the recently reported phase I/II TRINITI-1 study, the safety and efficacy of triplet therapy with the CDK4/6 inhibitor ribociclib (300 $\mathrm{mg}$ or $200 \mathrm{mg}$ ) plus everolimus (2.5 mg or $5 \mathrm{mg}$ ), along with exemestane (25 mg daily), was evaluated in men or postmenopausal women with HR+, HER2- MBC who progressed on prior CDK4/ 6 inhibitors and up to three lines of therapy ( $\geq 1$ ET and $\leq 1$ chemotherapy regimen). TRINITI-1 met its primary efficacy endpoint, demonstrating a clinical benefit rate of $41.1 \%$ at week 24 . Adverse events were consistent with the known safety profile of the individual drugs [94]. However, this is a single-arm study and further investigation is necessary. Ongoing research that aims to elucidate the intrinsic and acquired resistance mechanisms is also critical for the development of effective interventions following disease progression on CDK4/6 inhibitors. 


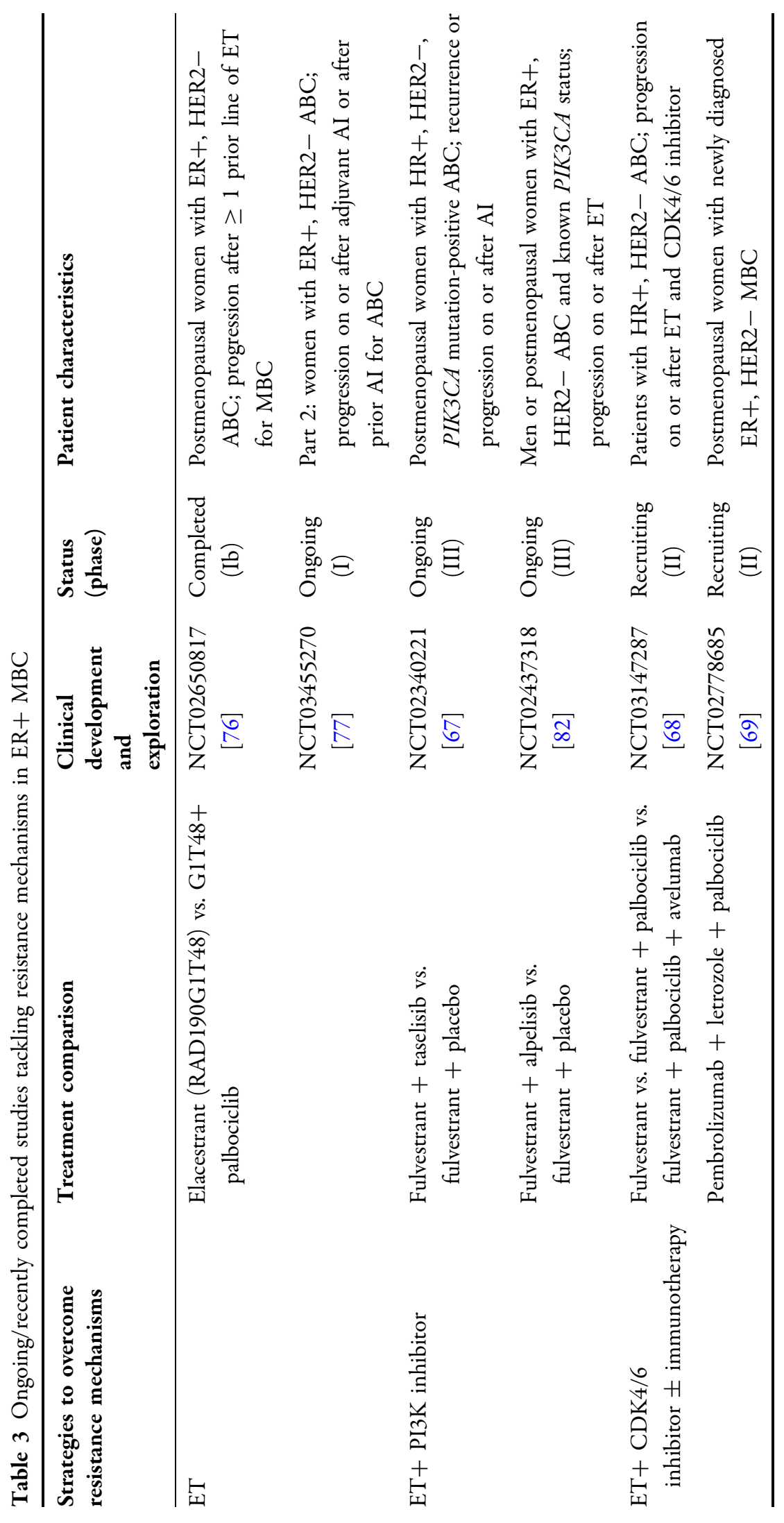




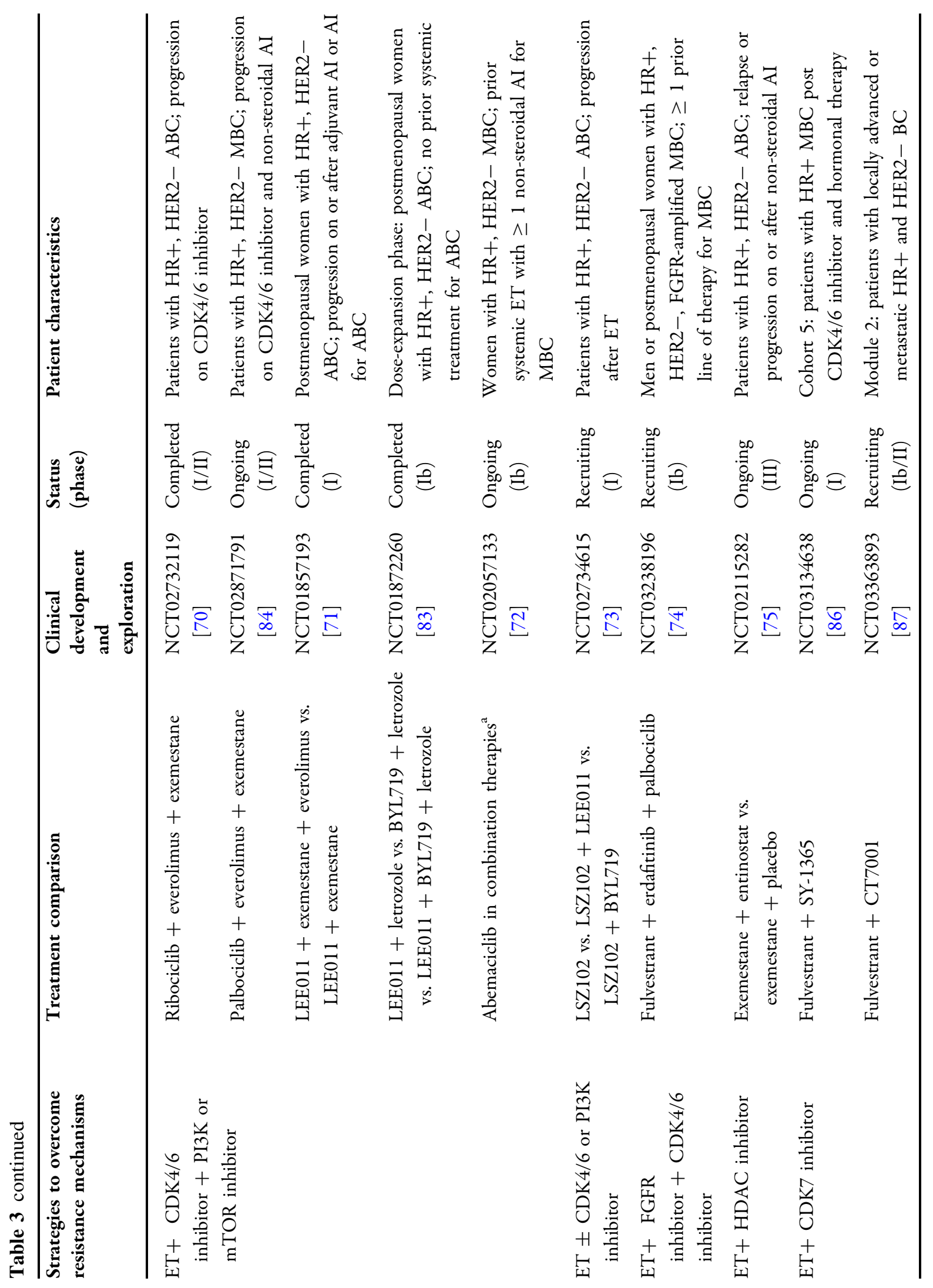




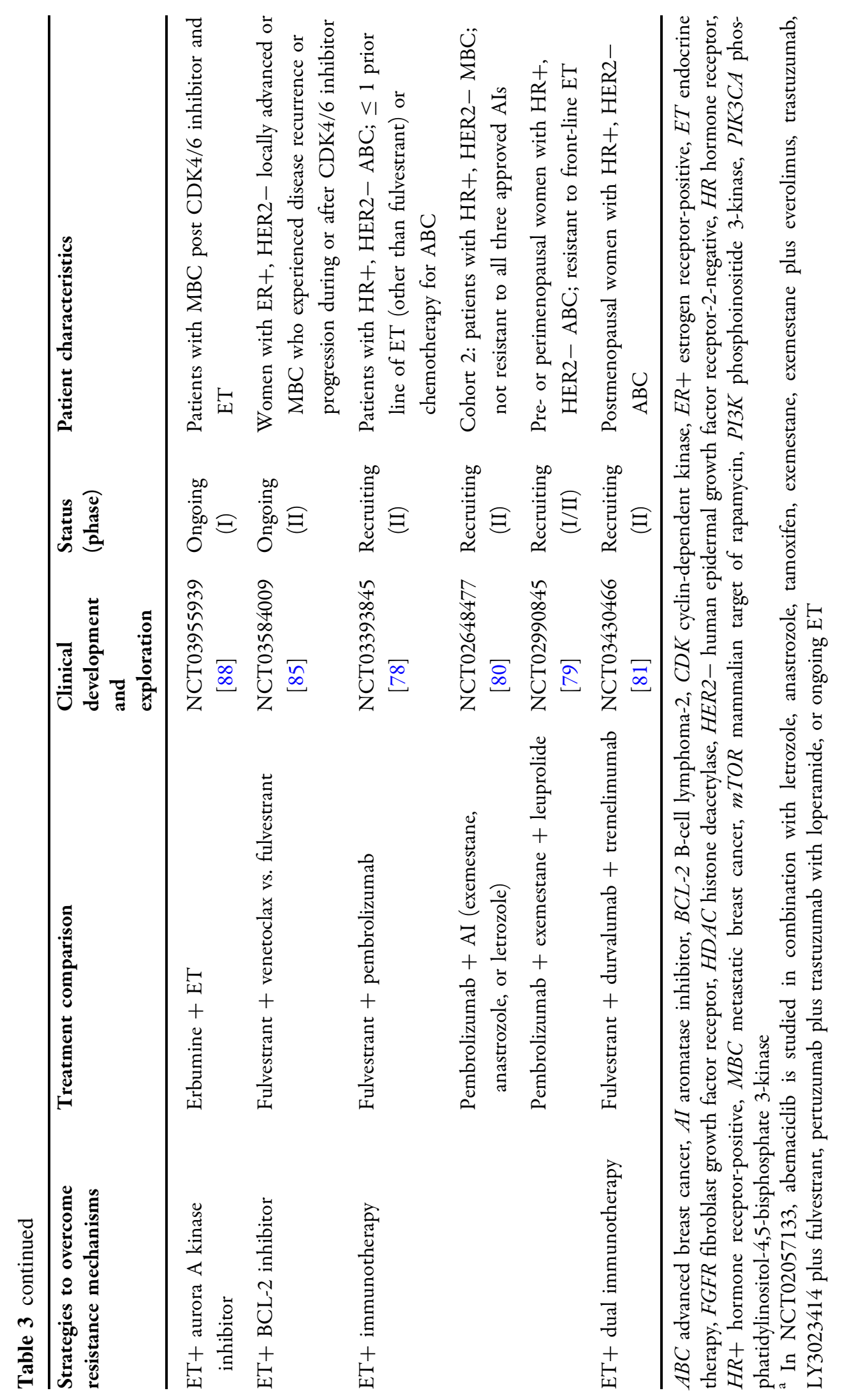




\section{WHAT ARE THE BIOMARKERS WITH CLINICAL UTILITY, OTHER THAN HORMONE RECEPTORS, IN ER+, HER2 - MBC?}

While many biomarkers are being evaluated in research arenas, those with potential therapeutic implications for clinical use are discussed below.

\section{ESR1 Mutation}

Of particular interest are the recurrent mutations in ESR1, which are highly prevalent in the tumors of women with ABC previously treated with AIs, but rarely occur in primary tumors $[16,95]$. These mutations cluster in the ligandbinding domain, leading to estrogen-independent constitutive ER activation and resistance to AIs [95]. Recurrent point mutations have been identified in the ESR1 ligand-binding domain, with common variants identified as D538G, Y537S, Y537N, and Y537C [95]. However, tumors bearing ESR1 mutations can retain relative sensitivity to tamoxifen and fulvestrant $[95,96]$. In a retrospective analysis of baseline plasma samples from the phase III SoFEA study, Fribbens et al. reported that median PFS significantly improved with fulvestrant-containing regimens versus the steroidal AI exemestane [hazard ratio 0.52 (95\% CI $0.30-0.92) ; P=0.02$ ] in patients with $\mathrm{HR}+$ ESR1 mutation-positive MBC; this difference was not significant in patients with wild-type ESR1 [hazard ratio 1.07 (95\% CI 0.68-1.67); $P=0.77$ ] [16]. These findings demonstrate that fulvestrant may be a more appropriate ET for the treatment of patients with tumors harboring ESR1 mutations. However, more effective SERDs are needed to optimize ET for ESR1-mutated BC, especially for the ESR1 Y537S mutation, which may be more resistant to fulvestrant than other known ESR1 mutations [97, 98]. Multiple new ER modulators, including lasofoxifene [99], LSZ102 [73], AZD9833 [100, 101], GDC-9545 [102], SAR439859 [103], G1T48 [77], and ZN-c5 [104] are undergoing clinical evaluation. Of these, an open-label phase II study is comparing lasofoxifene and fulvestrant for the treatment of pre- and postmenopausal women with $\mathrm{ER}+1$ HER2 - locally advanced or MBC with an acquired ESR1 mutation in patients who have disease progression on an AI in combination with a CDK4/6 inhibitor [99].

The potential impacts of ctDNA ESR 1 mutations on the outcome of patients treated with targeted therapies have been evaluated in clinical trials investigating mTOR inhibitors, as well as CDK4/6 inhibitors. In a secondary analysis of the BOLERO- 2 trial, $28.8 \%$ of evaluable patients had ESR1 mutations (Y537S and/or D538G) as detected by cell-free DNA analysis. These mutations were associated with aggressive biology and poor outcome [105]. Similar results were reported with the TRINITI-1 study, with shorter PFS observed in patients with ESR1 mutations compared with patients with wildtype ESR1, as detected by ctDNA genotyping [94]. However, exemestane was the ET partner in both studies. In the BOLERO-2 trial, benefit from addition of everolimus was see in both wild-type ESR1 and ESR1 D538G subgroups [105]. This study lacked sufficient numbers of patients in the ESR1 Y537S subgroup to draw conclusions on everolimus benefit for this subgroup. In the PALOMA-3 trial, the benefit of CDK4/6 inhibition with palbociclib was observed regardless of ESR1 mutation status [50]. However, positive selection of ESR1 Y537S mutation was observed on disease progression in both fulvestrant plus placebo and fulvestrant plus palbociclib treatment arms [106], which is consistent with previous studies demonstrating resistance of ESR1 Y537S mutation to fulvestrant [107]. The addition of CDK4/6 inhibitors to AI therapy does not appear to prevent emergence of ESR1 mutations [108].

\section{PIK3CA Mutation}

The PI3K-AKT-mTOR pathway is possibly the most commonly altered pathway in human cancers [53]. Mutations in the alpha catalytic subunit of PI3K (PIK3CA) are some of the most frequent genomic alterations noted in $\mathrm{HR}+\mathrm{BC}$ $[109,110]$. PIK3CA mutation status is an important predictive biomarker to identify 
patients suitable for treatment with alpelisib, a recently approved PI3K inhibitor, in combination with fulvestrant $[17,63]$. In addition to tumor testing, PIK3CA mutations can be detected by ctDNA testing, which offers a non-invasive option to screen patients for this therapeutic approach. In the SOLAR-1 study, a planned retrospective analysis demonstrated that alpelisib plus fulvestrant significantly improved median PFS versus fulvestrant monotherapy when PIK3CA mutations were detectable in plasma ctDNA (10.9 vs. 3.7 months) [64]. PIK3CA-mutational analysis in the SOLAR-1 study showed that greater reductions in the risk for disease progression were observed in patients with PIK3CA mutations detected in plasma ctDNA [hazard ratio 0.55 (95\% CI 0.39-0.79)] versus patients with PIK3CA mutations detected in tumor tissue samples [hazard ratio 0.65 (95\% CI 0.50-0.85); $P<0.001]$, highlighting the benefit of evaluating mutation status via liquid biopsy [64]. PIK3CA mutation status does not appear to predict benefit from CDK4/6 inhibitor therapy when used in combination with fulvestrant [49].

\section{HER2 Mutations}

Mutations in HER2 occur in approximately $2 \%$ of patients with BC overall, while, in lobular BC, HER2 mutations may occur more frequently [111]. A distinct mechanism of resistance to ET appears to be HER2 mutations [112]. Nayar et al. performed whole exome sequencing of metastatic biopsies to identify mechanisms of resistance in ER+ BC that are resistant to ET, including $\mathrm{AI}$, tamoxifen, and fulvestrant. Activating HER2 mutations were identified in 8 patients out of 168 samples [112]. Pre-existing mutations were not identified in treatmentnaïve primary tumor samples from 4 of 5 patients who were examined, which suggested that these mutations were acquired under selective pressure of ET. These mutations also appeared to be mutually exclusive with ESR1 mutations. Furthermore, the HER2 mutations exhibited resistance to tamoxifen, fulvestrant, and palbociclib, which was mitigated following treatment with a combination of ER-directed therapy and the irreversible HER2 kinase inhibitor, neratinib [112]. In another study, examination of data derived from 8 BC genomesequencing studies identified 25 patients with HER2 somatic mutations in HER2 gene amplification negative BC [111]. Of the 13 HER2 somatic mutations that were functionally characterized, 7 were determined to be activating mutations. Furthermore, whereas some of the HER2 mutations were resistant to lapatinib, a reversible HER2 inhibitor, all of the mutations were sensitive to neratinib, suggesting that drugs targeting HER2 may show clinical benefit in patients with HER2 somatic mutation-positive BC [111]. In line with these studies, a single-arm phase II trial was conducted to assess the clinical benefit rate of neratinib in heavily pre-treated patients with HER2-mutated nonamplified MBC [113]. Although this was a small, proof-of-concept study, the pre-defined primary efficacy endpoint was met, as the clinical benefit rate was 31\% (90\% CI 13-55). Additionally, in the SUMMIT basket trial evaluating neratinib in HER2- and HER3-mutated cancers, neratinib exhibited the greatest activity in HER2-mutant BC $(n=25$; ORR at Week 8 was 32\%) [114].

Neratinib in combination with fulvestrant in patients with ER+ HER2-mutated non-amplified $\mathrm{BC}$ is being evaluated in several trials [113]. In an amendment of the SUMMIT basket trial examining the combination of neratinib plus fulvestrant $(n=47)$, an ORR of $29.8 \%$ and clinical benefit rate of $46.8 \%$ was observed [vs. $36.4 \%$ and $36.4 \%$, respectively, in the ER-negative neratinib monotherapy subgroup $(n=11])$ and $17.4 \%$ and $30.4 \%$, respectively, in the ER+ neratinib monotherapy subgroup $(n=23)$ ] [115]. In a triplet therapy cohort of the SUMMIT basket trial, neratinib plus trastuzumab and fulvestrant achieved an overall response rate of $39 \%$ in 13 patients with HER2-mutant HR+ MBC [116]. In the phase II plasmaMATCH trial, neratinib in combination with fulvestrant showed clinical activity in patients with HER2mutated ER+ BC [117]. 


\section{Germline BRCA1 and BRCA2 Mutations}

Germline BRCA mutation status has therapeutic implications in the form of two recently approved poly (ADP-ribose) polymerase (PARP) inhibitors; olaparib and talazoparib [118, 119]. In the phase III OlympiAD study, patients with germline BRCA mutations and HER2- MBC who had received no more than two prior chemotherapy regimens for metastatic disease were randomly assigned to olaparib versus the physician's choice of a standard single-agent chemotherapy [120]. Of the 302 patients who underwent randomization, 205 patients were assigned to the olaparib group and 97 patients to the standard-therapy group. Median PFS was significantly longer with olaparib compared with the standard therapy [7.0 vs. 4.2 months; hazard ratio for PFS 0.58 (95\% CI 0.43-0.80); $P<0.001]$. The hazard ratio for PFS was better in the triple-negative subgroup [0.43 (95\% CI 0.29-0.63)] than in the HR+ subgroup [0.82 (95\% CI 0.55-1.26)]. The response rates were also higher in the olaparib group compared with the standard-therapy group (59.9 vs. $28.8 \%$ ) with lower rates of grade $\geq 3$ adverse events $(36.6 \%$ in the olaparib group vs. $50.5 \%$ in the standard-therapy group) [120]. In the final prespecified analysis of OS in the overall OlympiAD study population, median OS was not significantly different between treatment arms [19.3 months with olaparib and 17.1 months with standard therapy; hazard ratio 0.90 (95\% CI $0.66-1.23) ; P=0.513$; note that OlympiAD was not powered to show any difference in OS between treatment groups], and findings were consistent across pre-defined subgroups, including HR status [in the HR+ subgroup, median OS was 21.8 months with olaparib and 21.3 months with standard therapy; hazard ratio 0.86 (95\% CI 0.55-1.36); $P=$ not significant] [121]. In a similarly designed phase III, open-label, 2:1 randomized study, talazoparib was compared with the physician's choice of a standard single-agent chemotherapy in patients with $\mathrm{ABC}$ and germline BRCA mutations [122]. Median PFS was
8.6 months in the talazoparib group versus 5.6 months in the standard-therapy group [hazard ratio for disease progression or death 0.54 (95\% CI 0.41-0.71); $P<0.001]$. The hazard ratio for PFS was better in the HR+ subgroup [0.47 (95\% CI 0.32-0.71)] than in the triplenegative subgroup [0.60 (95\% CI 0.41-0.87)]. At the time of the interim analysis, the median hazard ratio for death was $0.76(95 \%$ CI $0.55-1.06 ; \quad P=0.11)$. Furthermore, the ORR determined by the investigators was greater for patients who received talazoparib compared with standard therapy (62.6 vs. $27.2 \%$, respectively). Hematologic grade 3-4 adverse events were observed in $55 \%$ of patients who were treated with talazoparib versus $38 \%$ of patients who were treated with standard therapy. Although the majority of non-hematologic adverse events were grade 1 in severity in patients who received talazoparib, non-hematologic grade 3 adverse events occurred in 32\% of patients in the talazoparib group compared with $38 \%$ of patients in the standard-therapy group. Significant improvements in patient-reported outcomes were observed in patients who received talazoparib compared with standard therapy [122].

\section{WHAT ARE THE EVOLVING PARADIGMS IN THE MANAGEMENT OF HR+, HER2- MBC?}

The role of precision medicine in oncology is evolving rapidly and is poised to play a very important role at every stage in the management of $\mathrm{MBC}$, with the goal of improving patient stratification and tailoring therapy based on druggable targets. In order to fully harness the potential of genomics in oncology, tumor DNA sequencing information is required to recognize biomarkers with prognostic and predictive significance for guiding future therapy and individualizing treatment. While tissue biopsy remains the current gold standard, genomic assessment from ctDNA is an emerging attractive tool since it is a blood-based assay 
compared with obtaining a tissue biopsy by invasive methods [123]. ctDNA testing is able to detect the active circulating tumor clone in the background of clonal heterogeneity that exists in metastatic disease [123, 124], and also offers the possibility of less-invasive longitudinal monitoring of disease genomic profiles [124]. Since bone is the most common site of distant metastasis in ER+ BCs [125], and 50\% of bone biopsy specimens have insufficient tissue for biomarker testing [126], this non-invasive testing will be an important option in this setting. If a liquid biopsy is planned, it is important to remember to do so at the time of progression. Concordance and discordance rates between the various platforms can be a challenge; however, further developments in this area may help solve this in future [124]. Genomic profiling tests are currently available to help guide appropriate treatment decisions [127]. In order to obtain regulatory drug approvals based on genomic alterations, innovative study designs such as basket and umbrella trials will have an increasingly important role to play [128]. The MATCH and TAPUR studies are examples of precision oncology clinical trials that explore treatment options based on molecular alterations $[129,130]$. Another important component to help advance precision oncology is to maintain large-scale repositories of real-world data, including clinical, genomic, molecular, and imaging information, along with patientreported outcomes, which can be shared among researchers. Real-world data repositories currently in progress include the Metastatic Breast Cancer Project and CancerLinQ. The increasing use of genomic and molecular testing in oncology generates an exponential volume of data. Therefore, there is a growing need to create novel models for sharing and integrating genomic, molecular, clinical, and patient-reported outcome data wherein artificial intelligence techniques, such as machine learning and deep learning, could play a key role. All these approaches make the future management of $\mathrm{MBC}$ challenging and promising at the same time.

\section{CONCLUSIONS}

Remarkable progress has been made in the management of $\mathrm{ER}+\mathrm{MBC}$ from agents that target the ER pathway to agents that target the various cellular mechanisms responsible for cancer survival and resistance to therapy. The data discussed above demonstrate the recent advancements in the field and the areas of ongoing research. In summary, the combination of ET and CDK4/6 inhibitor therapy is becoming a standard frontline therapy for ER+ MBC, with several clinical studies showing a survival benefit when a CDK4/6 inhibitor is combined with ET over ET alone [47, 51, 52]. However, the optimal sequence of treatment for patients who have had disease progression while receiving treatment with ET in combination with a CDK4/6 inhibitor is unknown. Sequencing therapies is an important aspect of managing $\mathrm{MBC}$, and the goal is to improve survival, balance disease control, and maintain quality of life. In the absence of rapidly progressing disease, extensive visceral disease burden, and endocrine resistance, ET alone or in combination with other targeted therapies are the preferred treatment options for subsequent lines of therapy for ER+ disease. Although there are some unique side effects related to ET and targeted therapies, they are generally tolerated better than chemotherapy, which paves the way for clinically meaningful advancements and a better quality of life. Options for subsequent lines of therapy will depend on which agents have previously been used; fulvestrant alone or in combination with CDK4/6 inhibitors may be considered, if not used previously. For patients who have progressed on ET with CDK 4/6 inhibitors, the combination of ET with everolimus and the combination of fulvestrant with alpelisib in patients with PIK3CA mutations are additional treatment recommendations.

For patients with disease progression following up to three sequential ET therapy regimens for advanced disease, chemotherapy may be considered [6]. The treatment approach may be similar for premenopausal patients once they have been rendered postmenopausal based on serum estradiol levels with ovarian suppression. 
The cornerstone of these recent advances lies in our increasing understanding of the molecular biology of BC and the utilization of cancer genomic tools. Genomic assessment with either tumor tissue or blood-based ctDNA assays are increasingly used. Drug approvals based on genomic markers of both somatic and germline mutations include alpelisib in combination with fulvestrant in PIK3CA-mutated ER+ MBC, and PARP inhibitors for BRCA1- and BRCA2mutated tumors. In addition, tumors with ESR1 and HER2 mutations are finding their place in clinical decision making.

The armamentarium available to manage MBC is increasing, with many promising novel agents and synergistic combination therapies on the horizon. Further advancements in our understanding of intra-tumoral heterogeneity, better predictive markers of sensitivity and resistance to selected therapies, innovative study designs, wider application of genomic tumor assessment tools, including ctDNA testing, and increased integration of genomic data in the clinical setting will help us make better treatment decisions for our patients. In addition, the use of artificial intelligence technology to aid complex decision making may be another potential tool to help treat MBC in the near future. However, the challenge of managing and applying the vast body of genomic data at a patient level, sharing the data for a wider understanding, and the economic impact of all of this has to be borne in mind.

\section{ACKNOWLEDGEMENTS}

Funding. This manuscript (including the journal's rapid service and open access fees) was funded by AstraZeneca LP, Wilmington, DE, USA and AstraZeneca, Cambridge, UK. The sponsor participated in manuscript review and the decision to submit the paper for publication.

Medical Writing and Editorial Assistance. The authors extend their thanks to Paula Davis, PhD, and Greg Tardie, PhD, of the Lockwood Group (Stamford, CT, USA) for providing medical writing support, and to Joan Hudson of the Lockwood Group (Stamford, CT, USA) for editorial support, both of which were in accordance with Good Publication Practice (GPP3) guidelines and funded by AstraZeneca LP (Wilmington, DE, USA). Medical writing support, under the direction of the authors, was also provided by Lauren McNally, MSc, CMC Connect, McCann Health Medical Communications, funded by AstraZeneca, Cambridge, UK, in accordance with GPP3 guidelines.

Authorship. All named authors meet the International Committee of Medical Journal Editors (ICMJE) criteria for authorship for this article, take responsibility for the integrity of the work as a whole, and have given their approval for this version to be published.

Disclosures. Gayathri Nagaraj received writing assistance funded by AstraZeneca. Cynthia $X$. Ma received research grants from Eisai, Novartis, Pfizer Inc, and Puma; consulting fees from AstraZeneca, Biovica, Eli Lilly, Merck, Myriad, Novartis, Pfizer Inc, Puma, Syndex, Tempus, and Total Health; fees for participation in review activities from Novartis; and writing assistance funded by AstraZeneca.

Compliance with Ethics Guidelines. This article is based on previously conducted studies and does not contain any studies with human participants or animals performed by any of the authors.

Open Access. This article is licensed under a Creative Commons Attribution-NonCommercial 4.0 International License, which permits any non-commercial use, sharing, adaptation, distribution and reproduction in any medium or format, as long as you give appropriate credit to the original author(s) and the source, provide a link to the Creative Commons licence, and indicate if changes were made. The images or other third party material in this article are included in the article's Creative Commons licence, unless indicated otherwise in a credit line to the material. If material is not included in the article's Creative Commons licence and your intended use is not permitted by statutory 
regulation or exceeds the permitted use, you will need to obtain permission directly from the copyright holder. To view a copy of this licence, visit http://creativecommons.org/licenses/by$\mathrm{nc} / 4.0 /$.

\section{REFERENCES}

1. Clarke R, Tyson JJ, Dixon JM. Endocrine resistance in breast cancer-an overview and update. Mol Cell Endocrinol. 2015;418(Pt 3):220-34.

2. Sørlie T, Perou CM, Tibshirani R, et al. Gene expression patterns of breast carcinomas distinguish tumor subclasses with clinical implications. Proc Natl Acad Sci USA. 2001;98:10869-74.

3. Anderson WF, Chatterjee N, Ershler WB, Brawley OW. Estrogen receptor breast cancer phenotypes in the Surveillance, Epidemiology, and End Results database. Breast Cancer Res Treat. 2002;76:27-36.

4. Milani A, Geuna E, Gloria M, Valabrega G. Overcoming endocrine resistance in metastatic breast cancer: current evidence and future directions. World J Clin Oncol. 2014;5:990-1001.

5. Yue W, Yager JD, Wang J-P, Jupe ER, Santen RJ. Estrogen receptor-dependent and independent mechanisms of breast cancer carcinogenesis. Steroids. 2013;78:161-70.

6. National Comprehensive Cancer Network. NCCN Clinical Practice Guidelines in Oncology: Breast Cancer v3.2020; 2020. https://www.nccn.org/ professionals/physician_gls/pdf/breast_blocks.pdf. Accessed 20 Apr 2020.

7. Files JA, Ko MG, Pruthi S. Managing aromatase inhibitors in breast cancer survivors: not just for oncologists. Mayo Clin Proc. 2010;85:560-6.

8. US Food and Drug Administration. Palbociclib highlights of prescribing information; 2018. https:// www.accessdata.fda.gov/drugsatfda_docs/label/2018/ 207103s007lbl.pdf. Accessed 06 Sep 2018.

9. US Food and Drug Administration. Ribociclib highlights of prescribing information; 2018. https:// www.accessdata.fda.gov/drugsatfda_docs/label/2018/ 209092s001lbl.pdf. Accessed 30 Jan 2019.

10. US Food and Drug Administration. Abemaciclib highlights of prescribing information; 2018. https:// www.accessdata.fda.gov/drugsatfda_docs/label/2018/ 208855s000lbl.pdf. Accessed 06 Sep 2018.
11. US Food and Drug Administration. Fulvestrant highlights of prescribing information; 2017. https:// www.accessdata.fda.gov/drugsatfda_docs/label/2017/ 021344s035lbl.pdf. Accessed 30 Jan 2019.

12. Mehta RS, Barlow WE, Albain KS, et al. Combination anastrozole and fulvestrant in metastatic breast cancer. N Engl J Med. 2012;367:435-44.

13. Mehta RS, Barlow WE, Albain KS, et al. Overall survival with fulvestrant plus anastrozole in metastatic breast cancer. N Engl J Med. 2019;380: 1226-34.

14. Wakeling AE, Dukes M, Bowler J. A potent specific pure antiestrogen with clinical potential. Cancer Res. 1991;51:3867-73.

15. Robertson JFR, Bondarenko IM, Trishkina E, et al. Fulvestrant $500 \mathrm{mg}$ versus anastrozole $1 \mathrm{mg}$ for hormone receptor-positive advanced breast cancer (FALCON): an international, randomised, doubleblind, phase 3 trial. Lancet. 2016;388:2997-3005.

16. Fribbens C, O'Leary B, Kilburn L, et al. Plasma ESR1 mutations and the treatment of estrogen receptorpositive advanced breast cancer. J Clin Oncol. 2016;34:2961-8.

17. Baselga J, Dent SF, Cortés J, et al. Phase III study of taselisib (GDC-0032) + fulvestrant (FULV) $v$ FULV in patients (pts) with estrogen receptor (ER)-positive, PIK3CA-mutant (MUT), locally advanced or metastatic breast cancer (MBC): primary analysis from SANDPIPER. J Clin Oncol. 2018;36(18_Suppl): Abstract LBA 1006.

18. André F, Ciruelos EM, Rubovszky G, et al. Alpelisib (ALP) + fulvestrant (FUL) for advanced breast cancer $(\mathrm{ABC})$ : results of the Phase 3 SOLAR-1 trial. ESMO 2018 Congress (19-23 Oct; Munich, Germany). Presentation LBA3_PR.

19. US Food and Drug Administration. FDA approves alpelisib for metastatic breast cancer; 2019. https:// www.fda.gov/drugs/resources-information-approveddrugs/fda-approves-alpelisib-metastatic-breast-cancer. Accessed 29 May 2019.

20. Nagaraj G, Ma C. Revisiting the estrogen receptor pathway and its role in endocrine therapy for postmenopausal women with estrogen receptorpositive metastatic breast cancer. Breast Cancer Res Treat. 2015;150:231-42.

21. Early Breast Cancer Trialists' Collaborative Group (EBCTCG). Aromatase inhibitors versus tamoxifen in early breast cancer: patient-level meta-analysis of the randomised trials. Lancet. 2015;386:1341-52. 
22. Bross PF, Cohen MH, Williams GA, Pazdur R. FDA drug approval summaries: fulvestrant. Oncologist. 2002;7:477-80.

23. Di Leo A, Jerusalem G, Petruzelka L, et al. Final overall survival: fulvestrant $500 \mathrm{mg}$ vs. $250 \mathrm{mg}$ in the randomized CONFIRM trial. J Natl Cancer Inst. 2014;106:djt337.

24. Di Leo A, Jerusalem G, Petruzelka L, et al. Results of the CONFIRM phase III trial comparing fulvestrant $250 \mathrm{mg}$ with fulvestrant $500 \mathrm{mg}$ in postmenopausal women with estrogen receptor-positive advanced breast cancer. J Clin Oncol. 2010;28:4594-600.

25. Robertson JF, Llombart-Cussac A, Rolski J, et al. Activity of fulvestrant $500 \mathrm{mg}$ versus anastrozole 1 $\mathrm{mg}$ as first-line treatment for advanced breast cancer: results from the FIRST study. J Clin Oncol. $2009 ; 27: 4530-5$.

26. Robertson JF, Lindemann JP, Llombart-Cussac A, et al. Fulvestrant $500 \mathrm{mg}$ versus anastrozole $1 \mathrm{mg}$ for the first-line treatment of advanced breast cancer: follow-up analysis from the randomized "FIRST" study. Breast Cancer Res Treat. 2012;136:503-11.

27. Ellis MJ, Llombart-Cussac A, Feltl D, et al. Fulvestrant $500 \mathrm{mg}$ versus anastrozole $1 \mathrm{mg}$ for the firstline treatment of advanced breast cancer: overall survival analysis from the Phase II FIRST study. J Clin Oncol. 2015;33:3781-7.

28. Llombart-Cussac A, Pérez-García JM, Bellet M, et al. PARSIFAL: a randomized, multicenter, open-label, phase II trial to evaluate palbociclib in combination with fulvestrant or letrozole in endocrine-sensitive patients with estrogen receptor $(\mathrm{ER})[+] / \mathrm{HER} 2[-]$ metastatic breast cancer. J Clin Oncol. 2020;38(15 Suppl):Abstract 1007.

29. Hanahan D, Weinberg RA. Hallmarks of cancer: the next generation. Cell. 2011;144:646-74.

30. Finn RS, Aleshin A, Slamon DJ. Targeting the cyclindependent kinases (CDK) 4/6 in estrogen receptorpositive breast cancers. Breast Cancer Res. 2016;18: 17.

31. Murphy CG, Dickler MN. The role of CDK4/6 inhibition in breast cancer. Oncologist. 2015;20: 483-90.

32. Cancer Genome Atlas Network. Comprehensive molecular portraits of human breast tumours. Nature. 2012;490:61-70.

33. Finn RS, Dering J, Conklin D, et al. PD 0332991, a selective cyclin D kinase 4/6 inhibitor, preferentially inhibits proliferation of luminal estrogen receptor-positive human breast cancer cell lines in vitro. Breast Cancer Res. 2009;11:R77.
34. Dickler MN, Tolaney SM, Rugo HS, et al. MONARCH 1, a phase II study of abemaciclib, a CDK4 and CDK6 inhibitor, as a single agent, in patients with refractory HR+/HER2-metastatic breast cancer. Clin Cancer Res. 2017;23:5218-24.

35. Finn RS, Martin M, Rugo HS, et al. Palbociclib and letrozole in advanced breast cancer. N Engl J Med. 2016;375:1925-36.

36. Hortobagyi GN, Stemmer SM, Burris HA, et al. Ribociclib as first-line therapy for HR-positive, advanced breast cancer. N Engl J Med. 2016;375: 1738-48.

37. Sledge GW Jr, Toi M, Neven P, et al. MONARCH 2: abemaciclib in combination with fulvestrant in women with HR+/HER2 - advanced breast cancer who had progressed while receiving endocrine therapy. J Clin Oncol. 2017;35:2875-84.

38. Yardley DA, Noguchi S, Pritchard KI, et al. Everolimus plus exemestane in postmenopausal patients with $\mathrm{HR}(+)$ breast cancer: BOLERO-2 final progression-free survival analysis. Adv Ther. 2013;30:870-84.

39. Goetz MP, Toi M, Campone M, et al. MONARCH 3: abemaciclib as initial therapy for advanced breast cancer. J Clin Oncol. 2017;35:3638-46.

40. Slamon DJ, Neven P, Chia S, et al. Phase III randomized study of ribociclib and fulvestrant in hormone receptor-positive, human epidermal growth factor receptor 2-negative advanced breast cancer: MONALEESA-3. J Clin Oncol. 2018;36:2465-72.

41. Loibl S, Turner NC, Ro J, et al. Palbociclib combined with fulvestrant in premenopausal women with advanced breast cancer and prior progression on endocrine therapy: PALOMA-3 results. Oncologist. 2017;22:1028-38.

42. Tripathy D, Im SA, Colleoni M, et al. Ribociclib plus endocrine therapy for premenopausal women with hormone-receptor-positive, advanced breast cancer (MONALEESA-7): a randomised phase 3 trial. Lancet Oncol. 2018;19:904-15.

43. Neven P, Rugo HS, Tolaney SM, et al. Abemaciclib for pre/perimenopausal women with $\mathrm{HR}+, \mathrm{HER} 2-$ advanced breast cancer. J Clin Oncol. 2018;36(18_ Suppl):Abstract 1002 .

44. Rugo HS, Finn RS, Diéras V, et al. Palbociclib plus letrozole as first-line therapy in estrogen receptorpositive/human epidermal growth factor receptor 2-negative advanced breast cancer with extended follow-up. Breast Cancer Res Treat. 2019;174: 719-29. 
45. Hortobagyi GN, Stemmer SM, Burris HA, et al. Updated results from MONALEESA-2, a phase III trial of first-line ribociclib plus letrozole versus placebo plus letrozole in hormone receptor-positive, HER2-negative advanced breast cancer. Ann Oncol. 2018;29:1541-7.

46. Im SA, Lu YS, Bardia A, et al. Overall survival with ribociclib plus endocrine therapy in breast cancer. N Engl J Med. 2019;381:307-16.

47. Slamon DJ, Neven P, Chia S, et al. Overall survival (OS) results of the Phase III MONALEESA-3 trial of postmenopausal patients (pts) with hormone receptor-positive $(\mathrm{HR}+)$, human epidermal growth factor 2-negative (HER2-) advanced breast cancer (ABC) treated with fulvestrant (FUL) \pm ribociclib (RIB). Ann Oncol. 2019;30(5_Suppl):Abstract LBA7_PR.

48. Gelmon KA, Cristofanilli M, Rugo HS, et al. Efficacy and safety of palbociclib plus endocrine therapy in North American women with hormone receptorpositive/human epidermal growth factor receptor 2-negative metastatic breast cancer. Breast J. 2020;26:368-75.

49. Cristofanilli M, Turner NC, Bondarenko I, et al. Fulvestrant plus palbociclib versus fulvestrant plus placebo for treatment of hormone-receptor-positive, HER2-negative metastatic breast cancer that progressed on previous endocrine therapy (PALOMA-3): final analysis of the multicentre, double-blind, phase 3 randomised controlled trial. Lancet Oncol. 2016;17:425-39.

50. Turner NC, Slamon DJ, Ro J, et al. Overall survival with palbociclib and fulvestrant in advanced breast cancer. N Engl J Med. 2018;379:1926-36.

51. Sledge GW Jr, Toi M, Neven P, et al. The effect of abemaciclib plus fulvestrant on overall survival in hormone receptor-positive, ERBB2-negative breast cancer that progressed on endocrine therapyMONARCH 2: a randomized clinical trial. JAMA Oncol. 2020;6:116-24.

52. Slamon DJ, Neven P, Chia S, et al. Overall survival with ribociclib plus fulvestrant in advanced breast cancer. N Engl J Med. 2020;382:514-24.

53. Liu P, Cheng H, Roberts TM, Zhao JJ. Targeting the phosphoinositide 3-kinase pathway in cancer. Nat Rev Drug Discov. 2009;8:627-44.

54. Miller TW, Balko JM, Arteaga CL. Phosphatidylinositol 3-kinase and antiestrogen resistance in breast cancer. J Clin Oncol. 2011;29:4452-61.

55. Ma CX, Crowder RJ, Ellis MJ. Importance of PI3kinase pathway in response/resistance to aromatase inhibitors. Steroids. 2011;76:750-2.
56. US Food and Drug Administration. Everolimus highlights of prescribing information; 2012. https:// www.accessdata.fda.gov/drugsatfda_docs/label/2012/ 022334s016lbl.pdf. Accessed 22 Nov 2018.

57. Piccart M, Hortobagyi GN, Campone M, et al. Everolimus plus exemestane for hormone-receptorpositive, human epidermal growth factor receptor2-negative advanced breast cancer: overall survival results from BOLERO-2. Ann Oncol. 2014;25: 2357-62.

58. Rugo HS, Seneviratne L, Beck JT, et al. Prevention of everolimus-related stomatitis in women with hormone receptor-positive, HER2-negative metastatic breast cancer using dexamethasone mouthwash (SWISH): a single-arm, phase 2 trial. Lancet Oncol. 2017;18:654-62.

59. Kornblum N, Zhao F, Manola J, et al. Randomized Phase II trial of fulvestrant plus everolimus or placebo in postmenopausal women with hormone receptor-positive, human epidermal growth factor receptor 2-negative metastatic breast cancer resistant to aromatase inhibitor therapy: results of PrE0102. J Clin Oncol. 2018;36:1556-63.

60. Krop IE, Mayer IA, Ganju V, et al. Pictilisib for oestrogen receptor-positive, aromatase inhibitorresistant, advanced or metastatic breast cancer (FERGI): a randomised, double-blind, placebo-controlled, phase 2 trial. Lancet Oncol. 2016;17: 811-21.

61. Baselga J, Im SA, Iwata H, et al. Buparlisib plus fulvestrant versus placebo plus fulvestrant in postmenopausal, hormone receptor-positive, HER2negative, advanced breast cancer (BELLE-2): a randomised, double-blind, placebo-controlled, phase 3 trial. Lancet Oncol. 2017;18:904-16.

62. Di Leo A, Johnston S, Lee KS, et al. Buparlisib plus fulvestrant in postmenopausal women with hormone-receptor-positive, HER2-negative, advanced breast cancer progressing on or after mTOR inhibition (BELLE-3): a randomised, double-blind, placebo-controlled, phase 3 trial. Lancet Oncol. 2018;19:87-100.

63. Dickler MN, Saura C, Richards DA, et al. A phase II study of the PI3K inhibitor taselisib (GDC-0032) combined with fulvestrant $(\mathrm{F})$ in patients (pts) with HER2-negative (HER2-), hormone receptor-positive $(\mathrm{HR}+)$ advanced breast cancer (BC). J Clin Oncol. 2016;34(15_Suppl):Abstract 520.

64. Juric D, Ciruelos E, Rubovszky G, et al. Alpelisib + fulvestrant for advanced breast cancer: subgroup analyses from the phase III SOLAR-1 trial. Cancer Res. 2019;79(4_Suppl):GS3-08. 
65. André F, Ciruelos E, Rubovszky G, et al. Alpelisib for PIK3CA-mutated, hormone receptor-positive advanced breast cancer. N Engl J Med. 2019;380: 1929-40.

66. Jones RH, Carucci M, Casbard AC, et al. Capivasertib (AZD5363) plus fulvestrant versus placebo plus fulvestrant after relapse or progression on an aromatase inhibitor in metastatic ER-positive breast cancer (FAKTION): a randomized, double-blind, placebo-controlled, phase II trial. J Clin Oncol. 2019;37(15_Suppl):Abstract 1016.

67. ClinicalTrials.gov. NCT02340221: a study of taselisib + fulvestrant versus placebo + fulvestrant in participants with advanced or metastatic breast cancer who have disease recurrence or progression during or after aromatase inhibitor therapy (SANDPIPER); 2015. https://clinicaltrials.gov/ct2/ show/NCT02340221. Accessed 05 Dec 2018.

68. ClinicalTrials.gov. NCT03147287: palbociclib after CDK and endocrine therapy (PACE); 2017. https:// clinicaltrials.gov/ct2/show/NCT03147287. Accessed 05 Dec 2018.

69. ClinicalTrials.gov. NCT02778685: pembrolizumab, letrozole, and palbociclib in treating postmenopausal patients with newly diagnosed metastatic stage IV estrogen receptor positive breast cancer; 2016. https://clinicaltrials.gov/ct2/show/ NCT02778685. Accessed 05 Dec 2018.

70. ClinicalTrials.gov. NCT02732119: study of ribociclib with everolimus + exemestane in HR+ HER2locally advanced/metastatic breast cancer post progression on CDK 4/6 inhibitor (TRINITI-1); 2016. https://clinicaltrials.gov/ct2/show/NCT02732119. Accessed 05 Dec 2018.

71. ClinicalTrials.gov. NCT01857193: phase Ib trial of LEE011 with everolimus (RAD001] and exemestane in the treatment of hormone receptor positive HER2 negative advanced breast cancer; 2013. https://clinicaltrials.gov/ct2/show/NCT01857193. Accessed 05 Dec 2018.

72. ClinicalTrials.gov. NCT02057133: a study of LY2835219 (abemaciclib) in combination with therapies for breast cancer that has spread; 2014. https://clinicaltrials.gov/ct2/show/NCT02057133. Accessed 05 Dec 2018.

73. ClinicalTrials.gov. NCT02734615: phase I/Ib trial of LSZ102 single agent or LSZ102 + LEE011 or LSZ102 + BYL719 in ER+ breast cancers; 2016. https:// clinicaltrials.gov/ct2/show/NCT02734615. Accessed 05 Dec 2018.

74. ClinicalTrials.gov. NCT03238196: fulvestrant, palbociclib and erdafitinib in ER+/HER2-/FGFR-amplified metastatic breast cancer; 2017. https://
clinicaltrials.gov/ct2/show/NCT03238196. Accessed 05 Dec 2018.

75. ClinicalTrials.gov. NCT02115282: exemestane with or without entinostat in treating patients with recurrent hormone receptor-positive breast cancer that is locally advanced or metastatic; 2014. https:// clinicaltrials.gov/ct2/show/NCT02115282. Accessed 05 Dec 2018.

76. ClinicalTrials.gov. NCT02650817: phase IB study to evaluate RAD1901 on the availability of estrogen receptor binding sites in metastatic breast cancer; 2016. https://clinicaltrials.gov/ct2/show/ NCT02650817. Accessed 05 Dec 2018.

77. ClinicalTrials.gov. NCT03455270: G1T48, an oral SERD, in ER-positive, HER2-negative advanced breast cancer; 2018. https://clinicaltrials.gov/ct2/ show/NCT03455270. Accessed 05 Dec 2018.

78. ClinicalTrials.gov. NCT03393845: study of pembrolizumab plus fulvestrant in hormone receptor positive, HER-2 negative advanced/metastatic breast cancer patients; 2018. https://clinicaltrials. gov/ct2/show/NCT03393845. Accessed 05 Dec 2018.

79. ClinicalTrials.gov. NCT02990845: pembrolizumab and exemestane/leuprolide in premenopausal $\mathrm{HR}+$ / HER2 - locally advanced or metastatic breast cancer (PEER); 2016. https://clinicaltrials.gov/ct2/show/ NCT02990845. Accessed 05 Dec 2018.

80. ClinicalTrials.gov. NCT02648477: pembrolizumab and doxorubicin hydrochloride or anti-estrogen therapy in treating patients with triple-negative or hormone receptor-positive metastatic breast cancer; 2016. https://clinicaltrials.gov/ct2/show/ NCT02648477. Accessed 05 Dec 2018.

81. ClinicalTrials.gov. NCT03430466: anti PD-L1 antibody + anti CTLA-4 antibody in combination with hormone therapy in patients with hormone receptor positive HER2-negative recurrent or metastatic breast cancer; 2018. https://clinicaltrials.gov/ct2/ show/NCT03430466. Accessed 05 Dec 2018.

82. ClinicalTrials.gov. NCT02437318: study assessing the efficacy and safety of alpelisib plus fulvestrant in men and postmenopausal women with advanced breast cancer which progressed on or after aromatase inhibitor treatment (SOLAR-1); 2015. https://clinicaltrials.gov/ct2/show/NCT02437318. Accessed 05 Dec 2018.

83. ClinicalTrials.gov. NCT01872260: study of LEE011, BYL719 and letrozole in advanced ER+ breast cancer; 2013. https://clinicaltrials.gov/ct2/show/ NCT01872260. Accessed 05 Dec 2018. 
84. ClinicalTrials.gov. NCT02871791: palbociclib with everolimus + exemestane in BC; 2016. https:// clinicaltrials.gov/ct2/show/NCT02871791. Accessed 05 Dec 2018.

85. ClinicalTrials.gov. NCT03584009: a phase II study comparing the efficacy of venetoclax + fulvestrant vs. fulvestrant in women with estrogen receptorpositive, Her2-negative locally advanced or metastatic breast cancer who experienced disease recurrence or progression during or after CDK4/6 inhibitor therapy; 2018. https://clinicaltrials.gov/ ct2/show/NCT03584009. Accessed 28 Oct 2019.

86. ClinicalTrials.gov. NCT03134638: study of SY-1365 in adult patients with advanced solid tumors; 2017. https://clinicaltrials.gov/ct2/show/NCT03134638. Accessed 28 Oct 2019.

87. ClinicalTrials.gov. NCT03363893: modular study to evaluate CT7001 alone in cancer patients with advanced malignancies; 2017. https://clinicaltrials. gov/ct2/show/NCT03363893. Accessed 28 Oct 2019.

88. ClinicalTrials.gov. NCT03955939: a study of LY3295668 erbumine in participants with breast cancer that has spread to other parts of the body; $2019 . \quad$ https://clinicaltrials.gov/ct2/show/ NCT03955939. Accessed 28 Oct 2019.

89. ClinicalTrials.gov. NCT03280563: a study of multiple immunotherapy-based treatment combinations in hormone receptor (HR)-positive human epidermal growth factor receptor 2 (HER2)-negative breast cancer (MORPHEUS HR+BC); 2017. https:// clinicaltrials.gov/ct2/show/NCT03280563. Accessed 17 Nov 2019.

90. Cardoso F, Senkus E, Costa A, et al. 4th ESO-ESMO International Consensus Guidelines for Advanced Breast Cancer (ABC 4) $\dagger$. Ann Oncol. 2018;29: 1634-57.

91. Baselga J, Campone M, Piccart M, et al. Everolimus in postmenopausal hormone-receptor-positive advanced breast cancer. N Engl J Med. 2012;366: 520-9.

92. ClinicalTrials.gov. NCT02738866: palbociclib with fulvestrant for metastatic breast cancer after treatment with palbociclib and an aromatase inhibitor; 2016. https://clinicaltrials.gov/ct2/show/ NCT02738866. Accessed 17 Nov 2019.

93. Dhakal A, Antony Thomas R, Levine EG, et al. Outcome of everolimus-based therapy in hormonereceptor-positive metastatic breast cancer patients after progression on palbociclib. Breast Cancer (Auckl). 2020;14:1178223420944864.
94. Bardia A, Hurvitz SA, DeMichele A, et al. Triplet therapy (continuous ribociclib, everolimus, exemestane) in HR+/HER2 - advanced breast cancer postprogression on a CDK4/6 inhibitor (TRINITI-1): efficacy, safety, and biomarker results. J Clin Oncol. 2019;37(15_Suppl):Abstract 1016.

95. Jeselsohn R, Buchwalter G, De Angelis C, Brown M, Schiff R. ESR1 mutations-a mechanism for acquired endocrine resistance in breast cancer. Nat Rev Clin Oncol. 2015;12:573-83.

96. Schiavon G, Hrebien S, Garcia-Murillas I, et al. Analysis of ESR1 mutation in circulating tumor DNA demonstrates evolution during therapy for metastatic breast cancer. Sci Transl Med. 2015;7: 313ra182.

97. De Santo I, McCartney A, Migliaccio I, Di Leo A, Malorni L. The emerging role of ESR1 mutations in luminal breast cancer as a prognostic and predictive biomarker of response to endocrine therapy. Cancers (Basel). 2019;11:1894.

98. Toy W, Weir H, Razavi P, et al. Activating ESR1 mutations differentially impact the efficacy of ER antagonists. Cancer Discov. 2017;7:277-87.

99. ClinicalTrials.gov. NCT03781063: evaluation of lasofoxifene versus fulvestrant in advanced or metastatic ER+/HER2- breast cancer with an ESR1 mutation; 2020. https://clinicaltrials.gov/ct2/show/ NCT03781063. Accessed 26 Aug 2020.

100. ClinicalTrials.gov. NCT03616587: study of AZD9833 alone or in combination in women with advanced breast cancer (SERENA-1); 2020. https:// clinicaltrials.gov/ct2/show/NCT03616587. Accessed 21 Sept 2020.

101. ClinicalTrials.gov. NCT04541433: a phase 1 study of AZD9833 in Japanese women with ER positive, HER2 negative advanced breast cancer); 2020. https://clinicaltrials.gov/ct2/show/NCT04541433. Accessed 21 Sept 2020.

102. ClinicalTrials.gov. NCT03332797: a study of GDC9545 alone or in combination with palbociclib and/ or luteinizing hormone-releasing hormone (LHRH) agonist in locally advanced or metastatic estrogen receptor-positive breast cancer; 2020. https:// clinicaltrials.gov/ct2/show/NCT03332797. Accessed 21 Sept 2020.

103. ClinicalTrials.gov. NCT03284957: phase $1 / 2$ study of SAR439859 single agent and in combination with palbociclib in postmenopausal women with estrogen receptor positive advanced breast cancer (AMEERA-1); 2020. https://clinicaltrials.gov/ct2/ show/NCT03284957. Accessed 21 Sept 2020. 
104. ClinicalTrials.gov. NCT03560531: a study of ZN-c5 in subjects with breast cancer; 2020. https:// clinicaltrials.gov/ct2/show/NCT03560531. Accessed 21 Sept 2020.

105. Chandarlapaty S, Chen D, He W, et al. Prevalence of ESR1 mutations in cell-free DNA and outcomes in metastatic beast cancer: a secondary analysis of the BOLERO-2 clinical trial. JAMA Oncol. 2016;2: 1310-5.

106. O'Leary B, Cutts RJ, Liu Y, et al. The genetic landscape and clonal evolution of breast cancer resistance to palbociclib plus fulvestrant in the PALOMA-3 trial. Cancer Discov. 2018;8:1390-403.

107. Martin L-A, Ribas R, Simigdala N, et al. Discovery of naturally occurring ESR1 mutations in breast cancer cell lines modelling endocrine resistance. Nat Commun. 2017;8:1865.

108. Gyanchandani R, Kota KJ, Jonnalagadda AR, et al. Detection of ESR1 mutations in circulating cell-free DNA from patients with metastatic breast cancer treated with palbociclib and letrozole. Oncotarget. 2017;8:66901-11.

109. Ellis MJ, Ding L, Shen D, et al. Whole-genome analysis informs breast cancer response to aromatase inhibition. Nature. 2012;486:353-60.

110. Mukohara T. PI3K mutations in breast cancer: prognostic and therapeutic implications. Breast Cancer (Dove Med Press). 2015;7:111-23.

111. Bose R, Kavuri SM, Searleman AC, et al. Activating HER2 mutations in HER2 gene amplification negative breast cancer. Cancer Discov. 2013;3:224-37.

112. Nayar U, Cohen O, Kapstad C, et al. Acquired HER2 mutations in $\mathrm{ER}(+)$ metastatic breast cancer confer resistance to estrogen receptor-directed therapies. Nat Genet. 2019;51:207-16.

113. Ma CX, Bose R, Gao F, et al. Neratinib efficacy and circulating tumor DNA detection of HER2 mutations in HER2 nonamplified metastatic breast cancer. Clin Cancer Res. 2017;23:5687-95.

114. Hyman DM, Piha-Paul SA, Won H, et al. HER kinase inhibition in patients with HER2- and HER3-mutant cancers. Nature. 2018;554:189-94.

115. Smyth LM, Piha-Paul SA, Won HH, et al. Efficacy and determinants of response to HER kinase inhibition in HER2-mutant metastatic breast cancer. Cancer Discov. 2020;10:198-213.

116. Wildiers H, Boni V, Saura C, et al. Neratinib + trastuzumab + fulvestrant for HER2-mutant, hormone receptor-positive, metastatic breast cancer: updated results from the phase 2 SUMMIT 'basket' trial. Cancer Res. 2020;80(4_Suppl):Abstract P1-19-08.

117. Wardley AM, Kilburn L, Kernaghan S, et al. Results from plasmaMATCH trial treatment Cohort B: a phase II trial of neratinib plus fulvestrant in ER positive breast cancer or neratinib alone in ER negative breast cancer in patients with ERBB2 (HER2) mutation identified via ctDNA screening (CRUK/15/010). San Antonio Breast Cancer Symposium (10-14 Dec 2019; San Antonio, TX, USA). Poster P1-19-07.

118. US Food and Drug Administration. Olaparib prescribing information; 2018. https://www. accessdata.fda.gov/drugsatfda_docs/label/2018/ 208558s006lbl.pdf. Accessed 31 Aug 2019.

119. US Food and Drug Administration. Talazoparib prescribing information; 2018. https:/www. accessdata.fda.gov/drugsatfda_docs/label/2018/ 211651s000lbl.pdf. Accessed 31 Aug 2019.

120. Robson M, Im SA, Senkus E, et al. Olaparib for metastatic breast cancer in patients with a germline BRCA mutation. N Engl J Med. 2017;377:523-33.

121. Robson ME, Tung N, Conte $P$, et al. OlympiAD final overall survival and tolerability results: olaparib versus chemotherapy treatment of physician's choice in patients with a germline BRCA mutation and HER2-negative metastatic breast cancer. Ann Oncol. 2019;30:558-66.

122. Litton JK, Rugo HS, Ettl J, et al. Talazoparib in patients with advanced breast cancer and a germline BRCA mutation. N Engl J Med. 2018;379: 753-63.

123. Diaz LA Jr, Bardelli A. Liquid biopsies: genotyping circulating tumor DNA. J Clin Oncol. 2014;32: 579-86.

124. Chae YK, Davis AA, Jain S, et al. Concordance of genomic alterations by next-generation sequencing in tumor tissue versus circulating tumor DNA in breast cancer. Mol Cancer Ther. 2017;16:1412-20.

125. Kennecke H, Yerushalmi R, Woods R, et al. Metastatic behavior of breast cancer subtypes. J Clin Oncol. 2010;28:3271-7.

126. Hilton JF, Amir E, Hopkins S, et al. Acquisition of metastatic tissue from patients with bone metastases from breast cancer. Breast Cancer Res Treat. 2011;129:761-5.

127. US Food and Drug Administration. FoundationOne CDx: summary of safety and effectiveness data; 2017. https://www.accessdata.fda.gov/cdrh_docs/ pdf17/P170019B.pdf. Accessed 28 Oct 2019. 
128. West HJ. Novel precision medicine trial designs: umbrellas and baskets. JAMA Oncol. 2017;3:423.

129. ClinicalTrials.gov. NCT02465060: targeted therapy directed by genetic testing in treating patients with advanced refractory solid tumors, lymphomas, or multiple myeloma (the MATCH screening trial); 2015. https://clinicaltrials.gov/ct2/show/NCT024 65060. Accessed 28 Oct 2019.
130. ClinicalTrials.gov. NCT02693535: TAPUR: testing the use of Food and Drug Administration (FDA) approved drugs that target a specific abnormality in a tumor gene in people with advanced stage cancer (TAPUR); 2016. https://clinicaltrials.gov/ct2/show/ NCT02693535. Accessed 28 Oct 2019. 